JOURNAL OF THE AMERICAN MATHEMATICAL SOCIETY

Volume 20, Number 1, January 2007, Pages 219-265

S 0894-0347(06)00531-5

Article electronically published on May 10, 2006

\title{
INTERMEDIATE SUBFACTORS WITH NO EXTRA STRUCTURE
}

\author{
PINHAS GROSSMAN AND VAUGHAN F. R. JONES
}

\section{INTRODUCTION}

Let $N \subseteq M$ be $\mathrm{II}_{1}$ factors with $[M: N]<\infty$. There is a "standard invariant" for $N \subseteq M$, which we shall describe using the planar algebra formalism of [19]. The vector spaces $P_{k}$ of $N-N$ invariant vectors in the $N-N$ bimodule $\otimes^{k} M$ admit an action of the operad of planar tangles as in 19] and 21. In more usual notation the vector space $P_{k}$ is the relative commutant $N^{\prime} \cap M_{k-1}$ in the tower $M_{k}$ of [16]. The conditional expectation $E_{N}$ from $M$ to $N$ is in $P_{2}$ and generates a planar subalgebra called the Temperley-Lieb algebra. In 4], Bisch and the second author studied the planar subalgebra of the $P_{k}$ generated by the conditional expectation onto a single intermediate subfactor $N \subseteq P \subseteq M$. The resulting planar algebra is called the Fuss-Catalan algebra and was generalised by Bisch and the second author to a chain of intermediate subfactors; see also [26]. These planar algebras are universal in that they are always planar subalgebras of the standard invariant for any subfactor possessing a chain of intermediate subfactors. If $P_{i} \subseteq P_{i+1}$ is the chain, there are no restrictions on the individual inclusions of $P_{i}$ in $P_{i+1}$. Moreover the existence of the Fuss-Catalan planar algebra together with a theorem of Popa in 32 allows one to construct a "free" increasing chain where the individual inclusions $P_{i} \subseteq P_{i+1}$ have "no extra structure"; i.e., their own standard invariants are just the Temperley-Lieb algebra. Thus the standard invariants for the $P_{i} \subseteq P_{i+1}$ are "decoupled" from the algebraic symmetries coming from the existence of a chain of intermediate subfactors.

In 33, Sano and Watatani considered the angle between two subfactors $P \subseteq M$ and $Q \subseteq M$, which we shall here define via the square of its cosine, namely the spectrum of the positive self-adjoint operator $E_{P} E_{Q} E_{P}\left(\right.$ on $\left.L^{2}(M)\right)$. In [25], Feng $\mathrm{Xu}$ and the second author proved that finiteness of the angle (as a substet of $[0,1]$ ) is equivalent to finiteness of the index of $P \cap Q$ in $M$. If we suppose that $P \cap Q$ is an irreducible finite index subfactor of $M$, then we might expect that the angle is

Received by the editors February 14, 2005.

2000 Mathematics Subject Classification. Primary 46L37.

Key words and phrases. Subfactors, planar algebras, intermediate subfactors.

The authors were supported in part by NSF Grant DMS04-01734; the second author was also supported by the Marsden fund UOA520 and the Swiss National Science Foundation.

(C)2006 American Mathematical Society Reverts to public domain 28 years from publication 
"quantized"; i.e., only a certain discrete countable family of numbers occurs (at least in a range close to 0 and $\pi / 2$ ). Determining these allowed angle values is becoming a significant question in the abstract theory of subfactors. This paper can be considered a first step in answering that question.

In [36], Watatani considered the lattice of intermediate subfactors for a finite index inclusion and showed that if the inclusion is irreducible the lattice is finite. He gave some constructions which allowed him to realise many simple finite lattices, but even for two lattices with only six elements, the question of their realisation as intermediate subfactor lattices remains entirely open.

The present paper grew out of an attempt by Dietmar Bisch and the second author to extend the methods of 4 to attack both the angle quantization and the intermediate lattice problems. The hope was to construct universal planar algebras depending only on the lattice of intermediate subfactors, and possibly the angles between them, and use Popa's theorem to construct subfactors realising the lattice and angle values. This project is probably sound, but it is hugely more difficult in the case where the lattice is not a chain or the angles are not all 0 or $\pi / 2$. The reason is very simple: the planar algebra generated by the conditional expectations can no longer be decoupled from the standard invariants of the elementary subfactor inclusions in the lattice. This is surprisingly easy to see. The spectral subspaces of $E_{P} E_{Q} E_{P}$ are $N-N$ bimodules contained in $P$ so that as soon as the angle operator has a significant spectrum the subfactor $N \subseteq M$ must have elements in its planar algebra that are not in the Temperley-Lieb subalgebra, a situation we shall refer to as having "extra structure" and which we will quantify using the notion of supertransitivity introduced in [23. In particular, if there is no extra structure the spectrum of $E_{P} E_{Q} E_{P}$ can consist of at most one number besides 0 and 1 . We will call the angle whose cosine is the square root of this number "the angle" between $P$ and $Q$. Or "dually" if $P Q P$ is not equal to all of $M$, then it is a nontrivial $P-P$ bimodule between $P$ and $M$ so that the inclusion $P \subseteq M$ must have extra structure.

Thus we are led to the following question: what are the possible pairs of subfactors $P$ and $Q$ in $M$ with $P \cap Q$ a finite index irreducible subfactor of $M$, for which the four elementary subfactors $N \subseteq P, N \subseteq Q, P \subseteq M$ and $Q \subseteq M$ all have no extra structure? More properly, since we are not trying to control the isomorphism type of the individual factors, one should ask what are the standard invariants that arise. One situation is rather easy to take care of: if the subfactors form a commuting cocommuting square in the sense of [33, then there is no obstruction. It was essentially observed by Sano and Watatani that in this case $E_{P}$ and $E_{Q}$ generate a tensor product of their individual Temperley-Lieb algebras. To realise any $N \subseteq P$ and $N \subseteq Q$ just take the tensor product $\mathrm{II}_{1}$ factors. However, if we assume that the subfactors either do not commute or do not cocommute, then we will show in this paper the following unexpected result.

\footnotetext{
$P \subset M$

Theorem 1.1. Suppose $\cup \quad \cup$ is a quadrilateral of subfactors with $N^{\prime} \cap M=$ $N \subset Q$

$\mathbb{C},[M: N]<\infty$ and no extra structure. Then either the quadrilateral commutes or one of the following two cases occurs.
} 
a) $[M: N]=6$ and $N$ is the fixed point algebra for an outer action of $S_{3}$ on $M$ with $P$ and $Q$ being the fixed point algebras for two transpositions in $S_{3}$. In this case the angle between $P$ and $Q$ is $\pi / 3$ and the full intermediate subfactor lattice is

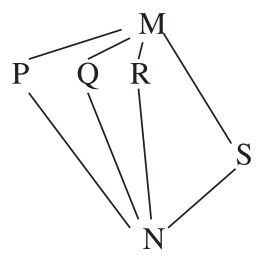

(Note that the dual of this quadrilateral is a commuting square.)

b) The subfactor $N$ is of depth $3,[M: N]=(2+\sqrt{2})^{2}$ and the planar algebra of $N \subseteq M$ is the same as that coming from the GHJ subfactor (see [12]) constructed from the Coxeter graph $D_{5}$ with the distinguished vertex being the trivalent one. Each of the intermediate inclusions has index $2+\sqrt{2}$ and the angle between $P$ and $Q$ is $\theta=\cos ^{-1}(\sqrt{2}-1)$. The principal graph of $N \subseteq M$ is

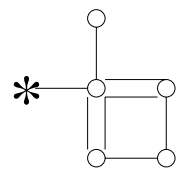

and the full intermediate subfactor lattice is

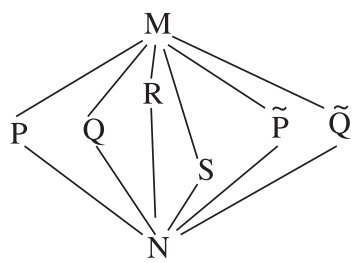

where the angle between $\tilde{P}$ and $\tilde{Q}$ is also $\theta$ but $P$ and $Q$ both commute with $\tilde{P}$ and $\tilde{Q}$. Moreover $[M: R]=[S: N]=2$ and $M, N, R$ and $S$ form a commuting cocommuting square. The planar algebra of $N \subseteq M$ is isomorphic to its dual-the planar algebra of $M \subseteq M_{1}$.

Note that from Ocneanu's paragroup point of view, $N$ is the fixed point algebra of an action of the paragroup given by the planar algebra on $M$. Thus if the ambient factor $M$ is hyperfinite, then Popa's theorem in 31 guarantees that the subfactors are unique up to an automorphism of $M$. Also note that it is a consequence of the theorem that any intermediate subfactor lattice with four elements and no extra structure is a commuting square.

Our methods rely heavily on planar algebras. Of crucial importance is the diagram discovered by Landau for the projection onto the product $P Q$. We give a proof of Landau's result and some general consequences. The uniqueness of the subfactor of index $6+4 \sqrt{2}$ mentioned in the theorem is proved using the "exchange relation" of 27. (the planar algebras have a very simple skein theory in the sense of [21]). The no-extra-structure hypothesis necessary for the theorem is in fact weaker than the one we have stated above. For a precise statement of the required supertransitivity, see Theorems 4.18 and 5.8 . 
After this paper was submitted, we learned from conversations with M. Izumi that he had found a way to obtain the restrictions on the index in our main theorem, and the uniqueness of the subfactor, by some bimodule arguments and [14. Also, the second author has proved that if $M$ is a $\mathrm{II}_{1}$ factor and $P$ and $Q$ are finite index subfactors, then the purely algebraic decomposition of $M$ as a $P-Q$ bimodule is the same as the decomposition of $L^{2}(M)$ as a $P-Q$ correspondence. This would allow one to avoid some awkward juggling of $L^{2}$ completions in discussions of the multiplication map between $N-N$ sub-bimodules of $M$ in this paper.

The authors would like to thank Dietmar Bisch and Zeph Landau for several fruitful discussions concerning this paper.

\section{BACKGROUND}

2.1. Bimodules. We recall some basic facts about bimodules over $\mathrm{II}_{1}$ factors. The treatment follows [3]. For more on this, look there and in [24].

Definition 2.1. Let $M$ be a $\mathrm{II}_{1}$ factor. A left $M$-module is a pair $(H, \pi)$ where $H$ is a Hilbert space and $\pi$ is a unital normal homomorphism from $M$ into the algebra of bounded operators on $H$. The dimension of $H$ over $M$, denoted $\operatorname{dim}_{M} H$, is the extended positive number given by the Murray-von Neumann coupling constant of $\pi(M)$. Let $M^{O P}$ be the opposite algebra of $M$ (i.e., the algebra with the same underlying vector space but with multiplication reversed). Then a right $M$-module is defined as a left $M^{O P}$-module. An $M-N$ bimodule is a triple $(H, \pi, \phi)$, where $H$ is a Hilbert space and $\pi$ and $\phi$ are normal unital homomorphisms from, respectively, $M$ and $N^{O P}$ into the algebra of bounded operators on $H$, such that $\pi(M)$ and $\phi\left(N^{O P}\right)$ commute. Such a bimodule is denoted by ${ }_{M} H_{N}$, or sometimes simply by $H$, if the action is understood. We write $m \xi n$ for $\pi(m) \phi(n) \xi$, where $m \in M$, $n \in N$, and $\xi \in H$.

There are obvious notions of submodules and direct sums. An $M-N$ bimodule is in particular both a left $M$-module and a left $N^{O P}$-module.

Definition 2.2. An $M-N$ bimodule is bifinite if $\operatorname{dim}_{M} H$ and $\operatorname{dim}_{N O P} H$ are both finite.

All bimodules will be assumed to be bifinite.

Definition 2.3. Let ${ }_{M} H_{N}^{1}$ and ${ }_{M} H_{N}^{2}$ be bimodules. The intertwiner space, denoted $\operatorname{Hom}_{M-N}\left(H^{1}, H^{2}\right)$, is the subspace of bounded operators from $H^{1}$ to $H^{2}$ consisting of those operators that commute with the bimodule action: $T \in \operatorname{Hom}_{M-N}\left(H^{1}, H^{2}\right)$ iff $T(m \xi n)=m(T \xi) n$ for all $m \in M, n \in N, \xi \in H^{1}$.

Example 2.4. Let $M$ be a $\mathrm{II}_{1}$ factor. $L^{2}(M)$ is the Hilbert space completion of $M$ with respect to the inner product induced by the unique normalized trace on $M$. Then $L^{2}(M)$ is an $M-M$ bimodule, and the left and right actions are simply the continuous extensions of ordinary left and right multiplication in $M$. If $P$ and $Q$ are subfactors of $M$, then $L^{2}(M)$ is a $P-Q$ bimodule by restriction, and it is bifinite iff the indices $[M: P]$ and $[M: Q]$ are finite.

Definition 2.5. Let ${ }_{M} H_{N}$ be a bimodule. There is a dense subspace $H^{0}$ of $H$, called the space of bounded vectors, defined by the rule that $\xi \in H^{0}$ iff the map $m \mapsto m \xi$ extends to a bounded operator from $L^{2}(M)$ to $H$. To each pair of bounded vectors $(\xi, \eta)$ there is associated an element of $M$, denoted $\langle\xi, \eta\rangle_{M}$, determined by the relation $\langle m \xi, \eta\rangle=\operatorname{tr}\left(m\langle\xi, \eta\rangle_{M}\right)$. 
Remark 2.6. It is in fact also true that $\xi \in H^{0}$ iff the map $n \mapsto n \xi$ extends to a bounded operator from $L^{2}(N)$ to $H$.

Remark 2.7. Let $M$ be a $\mathrm{II}_{1}$ factor, and consider $L^{2}(M)$ as a bimodule over a pair of finite index subfactors as in Example 2.0.5. Then $L^{2}(M)^{0}$ is simply the image of $M$ in $L^{2}(M)$.

There is a notion of fusion of bimodules due to Connes:

Definition 2.8. Let ${ }_{M} H_{N}$ and ${ }_{N} K_{P}$ be bimodules. There is an $M-P$ bimodule, denoted $\left({ }_{M} H_{N}\right) \otimes_{N}\left({ }_{N} K_{P}\right)$, called the relative tensor product, or fusion, of ${ }_{M} H_{N}$ and ${ }_{N} K_{P}$, which is characterized by the following property: there is a surjective linear map from the algebraic tensor product $H^{0} \odot K^{0}$ to $\left(\left({ }_{M} H_{N}\right) \otimes_{N}\left({ }_{N} K_{P}\right)\right)^{0}$, $\xi \otimes \eta \mapsto \xi \otimes_{N} \eta$ satisfying the following three conditions:

(i) $\xi n \otimes_{N} \eta=\xi \otimes_{N} n \eta$,

(ii) $m\left(\xi \otimes_{N} \eta\right) p=(m \xi) \otimes_{N}(\eta p)$,

(iii) $\left\langle\xi \otimes_{N} \eta, \xi^{\prime} \otimes_{N} \eta^{\prime}\right\rangle_{M}=\left\langle\xi\left\langle\eta, \eta^{\prime}\right\rangle_{N}, \xi^{\prime}\right\rangle_{M}$

(for all $m \in M, n \in N$, and $p \in P$ ).

Remark 2.9. Among the properties enjoyed by fusion are: it is distributive over direct sums, it is associative, and it is multiplicative in dimension, i.e.,

$$
\operatorname{dim}_{M}\left({ }_{M} H_{N} \otimes_{N}{ }_{N} K_{P}\right)=\left(\operatorname{dim}_{M} H\right)\left(\operatorname{dim}_{N} K\right) .
$$

Let $N \subset M$ be an inclusion of $\mathrm{II}_{1}$ factors with finite index. $L^{2}(N)$ can be identified with a subspace of $L^{2}(M)$. Let $e_{1}$ denote the corresponding projection on $L^{2}(M)$, and let $M_{1}$ be the von Neumann algebra generated by $M$ and $e_{1}$. Then $M_{1}$ is a $\mathrm{II}_{1}$ factor and $\left[M_{1}: M\right]=[M: N]$. This procedure is called the basic construction [16]. Recall that the space of bounded vectors in $L^{2}(M)$ can be identified with $M$. $e_{1}$ leaves this space invariant, inducing a trace-preserving conditional expectation of $M$ onto $N$.

Iterating the basic construction we get a sequence of projections $e_{1}, e_{2}, \ldots$ and a tower of algebras $M_{-1} \subset M_{0} \subset M_{1} \subset M_{2} \subset \ldots$, where $M_{-1}=N, M_{0}=M, e_{k}$ is the projection onto $L^{2}\left(M_{k-2}\right)$ in $B\left(L^{2}\left(M_{k-1}\right)\right)$, and $M_{k}$ is the von Neumann algebra generated by $M_{k-1}$ and $e_{k}$, for $k \geq 1$. Restricting the tower to those elements that commute with $N$, we get a tower of finite-dimensional algebras, called the tower of relative commutants $N^{\prime} \cap M_{k}$.

Each $L^{2}\left(M_{k}\right), k \geq 0$ is an $N-N$ bimodule, and

Proposition 2.10. $L^{2}\left(M_{k}\right) \cong L^{2}(M) \otimes_{N} \ldots \otimes_{N} L^{2}(M)$ ( $k+1$ factors) as an $N-N$ bimodule. Moreover, Hom ${ }_{N-N} L^{2}\left(M_{k}\right) \cong N^{\prime} \cap M_{2 k+1}$. So an $N-N$ bimodule decomposition of $\otimes_{N}^{k+1} L^{2}(M)$ corresponds to a decomposition of the identity in $N^{\prime} \cap M_{2 k+1}$. Under this correspondence projections in $N^{\prime} \cap M_{2 k+1}$ correspond to submodules of $\otimes_{N}^{k+1} L^{2}(M)$, minimal projections correspond to irreducible submodules (those that have no proper nonzero closed submodules), and minimal central projections to equivalence classes of irreducible submodules.

2.2. Planar algebras. In [19] a diagrammatic calculus was introduced as an axiomatisation and calculational tool for the standard invariant of a finite index subfactor. We will use it heavily in this paper, so we recall some of the essentials. The specific uses of the calculus in this paper make possible a couple of simplifying conventions for the pictures. 
In its most recent formulation in 23] a planar algebra $\mathfrak{P}$ consists of vector spaces $P_{k}^{ \pm}$indexed by a nonnegative integer $n$ and a sign + or - . For the planar algebra of a subfactor $N \subseteq M, P_{k}^{+}=N^{\prime} \cap M_{k-1}$ and $P_{k}^{-}=M^{\prime} \cap M_{k}$. The vector spaces $P_{k}^{ \pm}$form an algebra over the planar operad which means that there are multilinear maps between the $P_{k}^{ \pm}$indexed by planar tangles. A planar $k$-tangle $\mathfrak{T}$ consists of (i) the unit disc $D_{0}$ with $2 k$ distinguished boundary points, a finite number of disjoint interior discs $D_{j} \subset D_{0}$ for $k \geq 0$, each with an even number of distinguished boundary points, and smooth disjoint curves called strings, in $D_{0}$ meeting the $D_{j}$ exactly (transversally) in the distinguished boundary points;

(ii) a black and white shading of the regions of $\mathfrak{T}$ whose boundaries consist of the strings and the boundaries of the discs between the distinguished points. Regions of the tangle whose closures intersect are shaded different colours.

(iii) For each disc $D_{j}$ there is a choice of distinguished boundary interval between two adjacent distinguished points.

An example of a $k$-tangle is shown below (where we have used a $*$ near a boundary interval to indicate the chosen one).

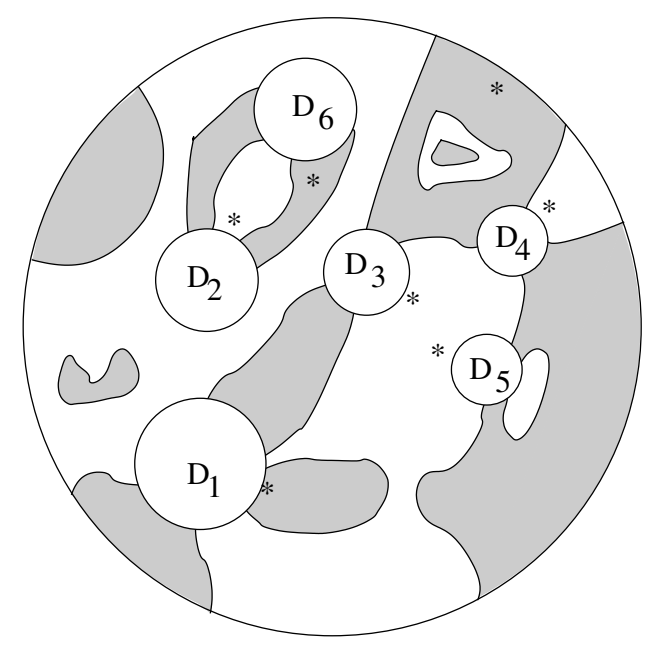

The multilinear map associated to the $k$-tangle $\mathfrak{T}$ goes from the product of the $P_{k_{j}}^{ \pm}$ for each internal disc, where $k$ is half the number of boundary points for $D_{j}$, to $P_{k}^{ \pm}$, the signs being chosen + if the distinguished boundary region is shaded and - if it is unshaded. The axioms of a planar algebra are that the multilinear maps be independent of isotopies globably fixing the boundary of $D_{0}$ and be compatible with the gluing of tangles in a sense made clear in [19]. To indicate the value of a tangle on its arguments one simply inserts the arguments in the internal discs. This notation for an element of $P_{k}$ is called a labelled tangle. For instance for $x \in P_{3}^{+}, y \in P_{2}^{+}$and $a, b, c, d \in P_{2}^{-}$, the labelled tangle below is the element of $P_{4}^{+}$obtained by applying the multilinear map defined by the tangle above to the 
elements $x, y, a, b, c, d$ according to the discs in which they are placed.

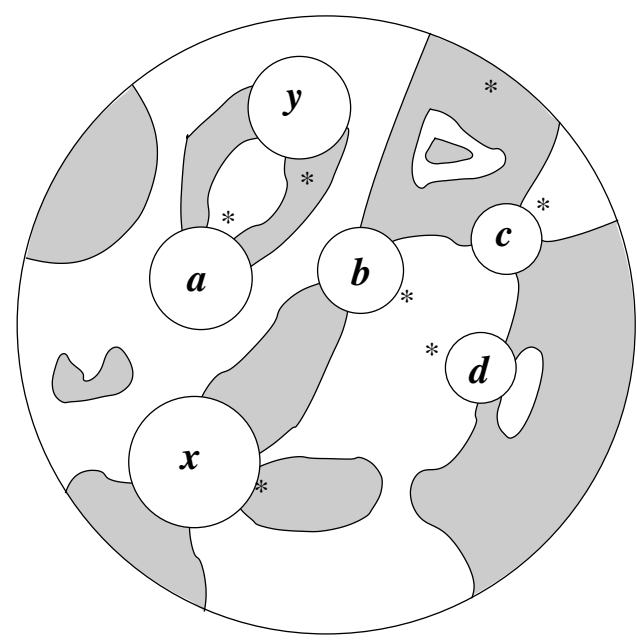

We refer to [19] for details on the meaning of various tangles and the fact that the standard invariant of a subfactor is a planar algebra. Recall that closed strings in a tangle can always be removed, each one counting for a multiplicative factor of the parameter $\delta$, which is the square root of the index for a subfactor planar algebra.

To avoid both the shading and the marking of the distinguished boundary interval we will adopt the following convention:

All discs will be replaced by rectangles called "boxes". The distinguished boundary points will be on a pair of opposite edges of each box, called the top and bottom. Labels will be well chosen letters which have a top and bottom which will allow us to say which edge is top and which is bottom. The distinguished interval will be supposed shaded and will always be between the first and second strings on the top of a box. This allows us to put elements of $P_{k}^{+}$in the boxes. So we further adopt the convention that if $t$ is in $P_{k}^{-}$, then it will be inserted at right angles to the top-bottom axis of its rectangle, which is to be interpreted as an internal disc whose distinguished (unshaded) interval is the edge of the rectangle to which the letter points upwards.

Thus the two diagrams below, with $a \in P_{2}^{-}$and $b, e \in P_{2}^{+}$represent the same thing according to our convention.
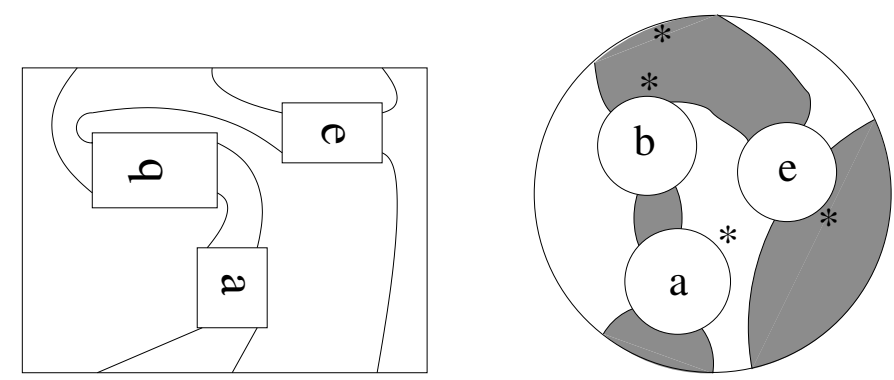
We will also from time to time simplify the diagrams further by suppressing the outside rectangle. Thus both of the above pictures are the same as the one below:

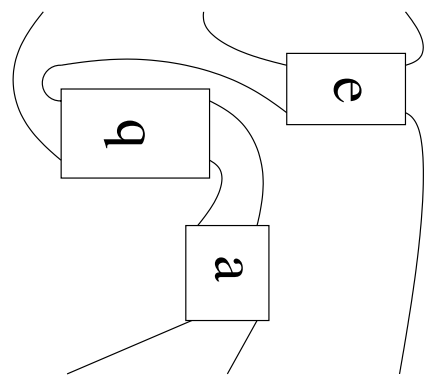

\section{Generalities}

3.1. Multiplication. Let $N \subset M$ be an irreducible inclusion of $\mathrm{II}_{1}$ factors with finite index, and suppose that $P$ and $Q$ are intermediate subfactors of this inclusion.

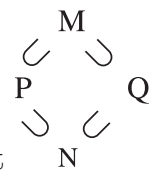

Following Sano and Watatani [33, we say that $\mathrm{N} \quad$ is a quadrilateral if $P \vee Q=$ $M$ and $P \wedge Q=N$. (There is no real loss of generality here since in any case we can restrict our attention to $P \vee Q$ and $P \wedge Q$.)

Proposition 3.1. The multiplication map from $P \otimes_{N} Q$ to $M$ extends to a surjective $N-N$ bimodule intertwiner from $L^{2}(P) \otimes_{N} L^{2}(Q)$ to $L^{2}(P Q)$.

Proof. The extension is simply (a scalar multiple of) the composition

$$
L^{2}(P) \otimes_{N} L^{2}(Q) \rightarrow L^{2}(M) \otimes_{N} L^{2}(M) \cong L^{2}\left(M_{1}\right) \rightarrow L^{2}(M),
$$

where the first map is the tensor product of the inclusions and the last map is the conditional expectation $e_{M}$.

Corollary 3.2. $L^{2}(P Q)$ is isomorphic as an $N-N$ bimodule to a submodule of $L^{2}(P) \otimes_{N} L^{2}(Q)$.

Remark 3.3. In a similar way, for any $k$, we can define a multiplication map from $\otimes_{N}^{k}\left(L^{2}(P) \otimes_{N} L^{2}(Q)\right)$ to $L^{2}\left((P Q)^{k}\right)$.

3.2. Comultiplication. Let $N \subset M$ be an irreducible inclusion of $\mathrm{II}_{1}$ factors with finite index. (Irreducible here means that $N^{\prime} \cap M \cong \mathbb{C}$.) Consider also the dual inclusion $M \subset M_{1}$.

Proposition 3.4. The first relative commutants $N^{\prime} \cap M_{1}$ and $M^{\prime} \cap M_{2}$ have the same vector space dimension, and the map $\phi: N^{\prime} \cap M_{1} \rightarrow M^{\prime} \cap M_{2}, a \mapsto$ $\delta^{3} E_{M^{\prime}}\left(a e_{2} e_{1}\right)$, is a linear isomorphism with inverse $a \mapsto \delta^{3} E_{M_{1}}\left(a e_{1} e_{2}\right)$, where $\delta=[M: N]^{\frac{1}{2}}$ and $E_{M^{\prime}}, E_{M_{1}}$ are the conditional expectations of $N^{\prime} \cap M_{2}$ onto $M^{\prime} \cap M_{2}$ and $N^{\prime} \cap M_{1}$ respectively.

Remark 3.5. In the planar picture, $\phi$ is simply

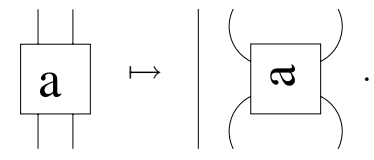


Pulling back the multiplication in $M^{\prime} \cap M_{2}$ via $\phi$ gives a second multiplication on $N^{\prime} \cap M_{1}$. Using the inner product given by the trace one may identify the vector space $N^{\prime} \cap M_{1}$ with its dual, and the second multiplication may thus be pulled back to the dual. If the depth of the subfactor is 2, then this multiplication on the dual induces a Hopf algebra structure on $N^{\prime} \cap M_{1}$, but in general this does not work. We will abuse terminology by calling the second multiplication on $N^{\prime} \cap M_{1}$ "comultiplication" and use the symbol o for it.

Definition 3.6. Let $a$ and $b$ be elements of $N^{\prime} \cap M_{1}$. Then $a \circ b=\phi^{-1}(\phi(b) \phi(a))=$ $\delta^{9} E_{M_{1}}\left(E_{M^{\prime}}\left(b e_{2} e_{1}\right) E_{M^{\prime}}\left(a e_{2} e_{1}\right) e_{1} e_{2}\right)$. Diagrammatically, $a \circ b$ is given by the picture

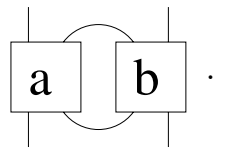

Remark 3.7. Dually, there is a comultiplication on $M^{\prime} \cap M_{2}$, also denoted by $\circ$, defined by pulling back the multipication via $\phi^{-1}$. Consequently, all of the formulas involving comultiplication have dual versions.

If $V$ is a vector subspace of $M$ which is closed under left and right multiplication by elements of $N$, then the closure of the image of $V$ in $L^{2}(M)$ is an $N-N$ submodule of $L^{2}(M)$, denoted by $L^{2}(V)$, and the corresponding projection (necessarily in $N^{\prime} \cap M_{1}$ ) by $e_{V}$. Conversely, any projection in $N^{\prime} \cap M_{1}$ is of the form $e_{V}$ for a strongly closed $N-N$ submodule $V$ of $M$, which is self-adjoint and multiplicatively closed iff $V$ is an intermediate subfactor. Bisch has shown that if $e$ is an arbitrary projection in $N^{\prime} \cap M_{1}$, then $e$ is of the form $e_{P}$ for an intermediate subfactor $P$ iff $e$ commutes with the modular conjugation on $L^{2}(M)$ and $e \circ e$ is a scalar multiple of $e$ [2. In that case $e$ is called a biprojection.

NOTATION: In the planar algebra pictures, discs will be labelled simply by $V$ instead of $e_{V}$.

Note that the set of biprojections inherits a partial order from the intermediate subfactor lattice. In particualr, $e_{1}=e_{N}=e_{N} e_{P}$ for any intermediate subfactor $P$.

If $N \subset P \subset M$ is an intermediate subfactor with biprojection $e_{P}$, then $\bar{P}=$ $\left\langle M, e_{P}\right\rangle$ is an intermediate subfactor of the dual inclusion $M \subset M_{1}$ with biprojection $e_{\bar{P}} \in M^{\prime} \cap M_{2}$.

Lemma 3.8. Suppose $e_{P}$ is a biprojection with dual biprojection $e_{\bar{P}}$. Then $E_{\bar{P}}\left(e_{1}\right)$ $=\delta^{-2} \operatorname{tr}\left(e_{P}\right)^{-1} e_{P}$.

Proof. Let $x$ and $y$ be elements of $M$. Then

$$
\begin{aligned}
\operatorname{tr}\left(e_{1} x e_{P} y\right) & =\operatorname{tr}\left(e_{1} e_{P} x e_{P} y\right)=\operatorname{tr}\left(e_{1} E_{P}(x) y\right)=\delta^{-2} \operatorname{tr}\left(E_{P}(x) y\right) \\
& =\delta^{-2} \operatorname{tr}\left(e_{P}\right)^{-1} \operatorname{tr}\left(e_{P} E_{P}(x) y\right)=\delta^{-2} \operatorname{tr}\left(e_{P}\right)^{-1} \operatorname{tr}\left(e_{P} x e_{P} y\right) .
\end{aligned}
$$

Lemma 3.9. With notation as above,

$$
\phi\left(e_{P}\right)=2 \sin \left(e_{P}\right) e_{\bar{P}} .
$$


Proof. We have

$$
\begin{aligned}
\phi^{-1}\left(e_{\bar{P}}\right) & =\delta^{3} E_{M_{1}}\left(e_{\bar{P}} e_{1} e_{2}\right)=\delta^{3} E_{M_{1}}\left(e_{\bar{P}} e_{1} e_{\bar{P}} e_{2}\right)=\delta^{3} E_{M_{1}}\left(E_{\bar{P}}\left(e_{1}\right) e_{2}\right) \\
& =\delta^{3} E_{\bar{P}}\left(e_{1}\right) E_{M_{1}}\left(e_{2}\right)=\delta^{-1} \operatorname{tr}\left(e_{P}\right)^{-1} e_{P} .
\end{aligned}
$$

Applying $\phi$ to both sides of the equation gives the result.

Let $P$ and $Q$ be intermediate subfactors of the inclusion $N \subset M$ with corresponding projections $e_{P}$ and $e_{Q}$. Then $\bar{P}=\left\langle M, e_{P}\right\rangle$ and $\bar{Q}=\left\langle M, e_{Q}\right\rangle$ are intermediate subfactors of the dual inclusion $M \subset M_{1}$, with corresponding projections $e_{\bar{P}}$ and $e_{\bar{Q}}$ in $M^{\prime} \cap M_{2}$.

The following result is due to Zeph Landau:

Theorem 3.10. (Landau)

$$
e_{P} \circ e_{Q}=\stackrel{+}{\mathbf{P}}=\delta \operatorname{tr}\left(e_{P} e_{Q}\right) e_{P Q}
$$

Proof. By Lemma 3.9 we have

$$
\begin{aligned}
e_{P} \circ e_{Q} & =\phi^{-1}\left(\phi\left(e_{Q}\right) \phi\left(e_{P}\right)\right)=\delta^{2} \operatorname{tr}\left(e_{Q}\right) \operatorname{tr}\left(e_{P}\right) \phi^{-1}\left(e_{\bar{Q}} e_{\bar{P}}\right) \\
& =\delta^{5} \operatorname{tr}\left(e_{Q}\right) \operatorname{tr}\left(e_{P}\right) E_{M_{1}}\left(e_{\bar{Q}} e_{\bar{P}} e_{1} e_{2}\right) .
\end{aligned}
$$

By a small abuse of notation, we shall identify $M$ with its image in $L^{2}(M)$. Let $x \in M$. For any $a \in N^{\prime} \cap M_{1}$, we have $a(x)=\delta^{2} E_{M}\left(a x e_{1}\right)$. In particular,

$$
\begin{aligned}
e_{P} \circ e_{Q}(x) & =\delta^{5} \operatorname{tr}\left(e_{P}\right) \operatorname{tr}\left(e_{Q}\right) E_{M_{1}}\left(e_{\bar{Q}} e_{\bar{P}} e_{1} e_{2}\right)(x) \\
& =\delta^{7} \operatorname{tr}\left(e_{P}\right) \operatorname{tr}\left(e_{Q}\right) E_{M}\left(e_{\bar{Q}} e_{\bar{P}} e_{1} e_{2} x e_{1}\right) .
\end{aligned}
$$

Let $y$ be another element of $M$. Then

$$
\begin{aligned}
\operatorname{tr}\left(e_{\bar{Q}} e_{\bar{P}} e_{1} e_{2} x e_{1} y\right) & =\operatorname{tr}\left(e_{\bar{P}} e_{1} e_{\bar{P}} e_{2} x e_{2} e_{\bar{Q}} e_{1} e_{\bar{Q}} y\right) \\
& =\delta^{-4} \operatorname{tr}\left(e_{P}\right)^{-1} \operatorname{tr}\left(e_{Q}\right)^{-1} \operatorname{tr}\left(e_{P} e_{2} x e_{Q} y\right) \\
& =\delta^{-6} \operatorname{tr}\left(e_{P}\right)^{-1} \operatorname{tr}\left(e_{Q}\right)^{-1} \operatorname{tr}\left(e_{P} x e_{Q} y\right) .
\end{aligned}
$$

Thus $E_{M}\left(e_{\bar{Q}} e_{\bar{P}} e_{1} e_{2} x e_{1}\right)=\delta^{-6} \operatorname{tr}\left(e_{P}\right)^{-1} \operatorname{tr}\left(e_{Q}\right)^{-1} E_{M}\left(e_{P} x e_{Q}\right)$ and $e_{P} \circ e_{Q}(x)=$ $\delta E_{M}\left(e_{P} x e_{Q}\right)$.

So if $x=p q$, with $p \in P$ and $q \in Q$, then $e_{P} \circ e_{Q}(x)=\delta E_{M}\left(e_{P} x e_{Q}\right)=$ $\delta E_{M}\left(e_{P} e_{Q}\right) x=\delta \operatorname{tr}\left(e_{P} e_{Q}\right)(x)$.

To finish the proof, it suffices to show that $e_{P} \circ e_{Q}$ vanishes on the orthogonal complement of $L^{2}(P Q)$, or equivalently, that if $\operatorname{tr}(x q p)=0$ for all $p \in$ $P, q \in Q$, then $E_{M}\left(e_{P} x e_{Q}\right)=0$. So suppose $\operatorname{tr}(x q p)=0$ for all $p \in P, q \in Q$. Let $\left\{p_{i}\right\},\left\{q_{j}\right\}$ be Pimsner-Popa bases over $N$ for $P$ and $Q$, respectively. Then $e_{P}=\sum p_{i} e_{1} p_{i}^{*}$ and $e_{Q}=\sum q_{i} e_{N} q_{i}^{*}$. Suppose $y \in M$. For any $i, j$, we have $\operatorname{tr}\left(p_{i} e_{1} p_{i}^{*} x q_{j} e_{1} q_{j}^{*} y\right)=\delta^{-2} \operatorname{tr}\left(p_{i}^{*} x q_{i} E_{N}\left(q_{j}^{*} y p_{i}\right)\right)=\delta^{-2} \operatorname{tr}\left(x q_{i} E_{N}\left(q_{j}^{*} y p_{i}\right) p_{i}^{*}\right)=0$. This implies that $E_{M}\left(e_{P} x e_{Q}\right)=0$.

Corollary 3.11. $e_{P Q}(M)=P Q$.

Proof. From the proof we have $e_{P Q}(x)=\operatorname{tr}\left(e_{P} e_{Q}\right)^{-1} E_{M}\left(e_{P} x e_{Q}\right)$. Moreover $e_{P}=$ $\sum p_{i} e_{1} p_{i}^{*}$ and $e_{Q}=\sum q_{i} e_{N} q_{i}^{*}$ with the same notation as in Theorem 3.10. We see that $e_{P Q}(M) \subset P Q$. 
Corollary 3.12. $P Q$ is strongly closed in $M$.

Proof. Since $e_{P Q}$ is strongly continuous and the identity on $P Q, e_{P Q}$ is the identity on the strong closure of $P Q$.

Lemma 3.13. Let $a \in N^{\prime} \cap M_{1}$. Then

$$
\mathrm{a}_{\mathrm{a}}=\stackrel{\mathrm{a}}{\mathrm{a}}=\delta \operatorname{tr}(a)
$$

Proof. Labelled tangles with two boundary points are elements of $N^{\prime} \cap M$, which by irreducibility must be scalars. So

$$
\mathbf{a}^{a}=\operatorname{tr}(\overrightarrow{\mathbf{a}})=\delta^{-1} \operatorname{tr}(
$$

One corollary of Theorem 3.10 is the following multiplication formula:

Proposition 3.14. $\operatorname{tr}\left(e_{P Q}\right) \operatorname{tr}\left(e_{P} e_{Q}\right)=\operatorname{tr}\left(e_{P}\right) \operatorname{tr}\left(e_{Q}\right)$.

Proof.

$$
\begin{aligned}
& \delta \operatorname{tr}\left(e_{P Q}\right) \operatorname{tr}\left(e_{P} e_{Q}\right)=\operatorname{tr}(\stackrel{\mathrm{P} \quad \mathrm{Q}}{\mathrm{Q}}) \\
& =\delta^{-2}\left(\left[\begin{array}{l}
\mathbf{P} \\
\mathbf{Q}
\end{array}\right)=\delta^{-2}\left(\left[\begin{array}{l|l}
\mathbf{P} & \mathbf{Q}
\end{array}\right)(\square)\right.\right. \\
& =\delta \operatorname{tr}\left(e_{P}\right) \operatorname{tr}\left(e_{Q}\right) \text {. }
\end{aligned}
$$

Corollary 3.15. $\operatorname{tr}\left(e_{P Q}\right)=\operatorname{tr}\left(e_{Q P}\right)$.

And another trace formula:

Lemma 3.16. $\operatorname{tr}\left(e_{P} e_{Q}\right)=\frac{1}{\operatorname{dim}_{M} L^{2}(\bar{P} \bar{Q})}$.

Proof. $\frac{1}{\operatorname{dim}_{M} L^{2}(\bar{P} \bar{Q})}=\frac{1}{\delta^{2} \operatorname{tr}\left(e_{\bar{P} \bar{Q}}\right)}=\frac{\operatorname{tr}\left(e_{\bar{P}} e_{\bar{Q}}\right)}{\delta^{2} \operatorname{tr}\left(e_{\bar{P}}\right) \operatorname{tr}\left(e_{\bar{Q}}\right)}$, by Proposition 3.14. By Lemma 3.9.

$$
e_{\bar{P}}=\frac{1}{\delta \operatorname{tr}\left(e_{P}\right)} \phi\left(e_{P}\right)
$$


so we have

$$
\begin{aligned}
\frac{1}{\operatorname{dim}_{M} L^{2}(\bar{P} \bar{Q})} & =\frac{1}{\delta^{4} \operatorname{tr}\left(e_{\bar{P}}\right) \operatorname{tr}\left(e_{\bar{Q}}\right) \operatorname{tr}\left(e_{P}\right) \operatorname{tr}\left(e_{Q}\right)} \operatorname{tr}\left(\phi\left(e_{P}\right) \phi\left(e_{Q}\right)\right) \\
& =\operatorname{tr}\left(\phi\left(e_{P}\right) \phi\left(e_{Q}\right)\right) \\
& =\delta^{-2}
\end{aligned}
$$

On the other hand,

$$
\begin{aligned}
\mathrm{P} & =\delta^{3} \operatorname{tr}\left(e_{1}\left(e_{P} \circ e_{Q}\right) e_{1}\right) \\
& =\delta^{4} \operatorname{tr}\left(e_{P} e_{Q}\right) \operatorname{tr}\left(e_{1}\right)=\delta^{2} \operatorname{tr}\left(e_{P} e_{Q}\right) .
\end{aligned}
$$

Combining these two equations gives the result.

We mention one more formula which we will need later.

Lemma 3.17. $\operatorname{tr}\left(e_{P Q} e_{Q P}\right)=\left(\delta \operatorname{tr}\left(e_{P Q}\right)\right)^{2} \operatorname{tr}\left(\left(e_{\bar{P}} e_{\bar{Q}} e_{\bar{P}}\right)^{2}\right)$.

Proof. By Theorem 3.10 ,

$$
\begin{aligned}
\operatorname{tr}\left(e_{P Q} e_{Q P}\right) & =\frac{\operatorname{tr}\left(\left(e_{P} \circ e_{Q}\right)\left(e_{Q} \circ e_{P}\right)\right)}{\left(\delta \operatorname{tr}\left(e_{P} e_{Q}\right)\right)\left(\delta \operatorname{tr}\left(e_{Q} e_{P}\right)\right)} \\
& =\frac{1}{\delta^{4}\left(\operatorname{tr}\left(e_{P} e_{Q}\right)\right)^{2}}
\end{aligned}
$$

On the other hand, by Lemma 3.9.

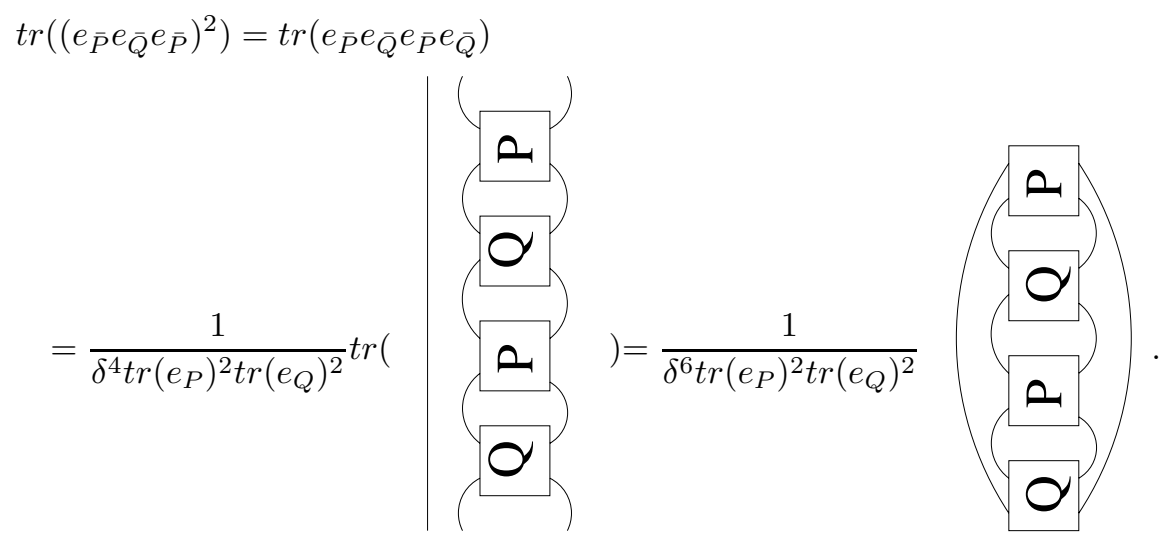


By [2] the 2-box for a biprojection is invariant under rotation by $\pi$, so the two trace pictures are the same. Combining these two equations then gives

$$
\operatorname{tr}\left(e_{P Q} e_{Q P}\right)=\delta^{2} \frac{\operatorname{tr}\left(e_{P}\right)^{2} \operatorname{tr}\left(e_{Q}\right)^{2}}{\operatorname{tr}\left(e_{P} e_{Q}\right)^{2}} \operatorname{tr}\left(\left(e_{\bar{P}} e_{\bar{Q}} e_{\bar{P}}\right)^{2}\right),
$$

which by Proposition 3.14 equals $\left(\delta \operatorname{tr}\left(e_{P Q}\right)\right)^{2} \operatorname{tr}\left(\left(e_{\bar{P}} e_{\bar{Q}} e_{\bar{P}}\right)^{2}\right)$.

3.3. Commuting and cocommuting quadrilaterals. Following Sano and Watatani [33] we consider the condition that a quadrilateral forms a commuting square, which means that $e_{P} e_{Q}=e_{Q} e_{P}$. A quadrilateral is called a cocommuting square if the dual quadrilateral is a commuting square.

Lemma 3.18. Let $\stackrel{\mathrm{P}}{\circlearrowright} \mathrm{N}_{\mathrm{Q}}^{\mathrm{M}}$ be a quadrilateral of $\mathrm{II}_{1}$ factors, where $N \subset M$ is an irreducible finite-index inclusion. Consider the multiplication map of Proposition 3.1 from $L^{2}(P) \otimes_{N} L^{2}(Q)$ to $L^{2}(P Q)$. The quadrilateral commutes iff this map is injective and cocommutes iff the map is surjective.

Proof. The quadrilateral commutes iff $e_{P} e_{Q}=e_{Q} e_{P}$ iff $e_{P} e_{Q}=e_{N}$. By Proposition 3.14 this is equivalent to $\frac{1}{[M: N]}=\operatorname{tr}\left(e_{N}\right)=\operatorname{tr}\left(e_{P} e_{Q}\right)=\frac{\operatorname{tr}\left(e_{P}\right) \operatorname{tr}\left(e_{Q}\right)}{\operatorname{tr}\left(e_{P Q}\right)}$, or

$$
\begin{aligned}
\operatorname{dim}_{N} L^{2}(P Q) & =[M: N] \operatorname{tr}\left(e_{P Q}\right)=[M: N]^{2} \operatorname{tr}\left(e_{P}\right) \operatorname{tr}\left(e_{Q}\right) \\
& =\operatorname{dim}_{N} L^{2}(P) \cdot \operatorname{dim}_{N} L^{2}(Q)=\operatorname{dim}_{N} L^{2}(P) \otimes_{N} L^{2} Q .
\end{aligned}
$$

But by Corollary [3.2, $L^{2}(P Q)$ is isomorphic to a submodule of $L^{2}(P) \otimes_{N} L^{2}(Q)$, so the two have the same $N$-dimension iff they are in fact isomorphic, which is equivalent to the injectivity of the multiplication map.

The quadrilateral cocommutes iff $e_{\bar{P} \bar{Q}}=e_{\bar{Q} \bar{P}}=e_{M}$. By Lemma 3.16, this is equivalent to $\operatorname{dim}_{N} L^{2}(P Q)=\frac{1}{\operatorname{tr}\left(e_{\bar{P} \bar{Q}}\right)}=\frac{1}{\operatorname{tr}\left(e_{M}\right)}=\operatorname{dim}_{N} L^{2}(M)$, which is equivalent to $L^{2}(P Q)=L^{2}(M)$.

Corollary 3.19. The quadrilateral commutes iff

$$
\operatorname{dim}_{N} L^{2}(P Q)=\operatorname{dim}_{N}\left(L^{2}(P) \otimes_{N} L^{2}(Q)\right)=[P: N][Q: N] .
$$

Corollary 3.20. The quadrilateral cocommutes iff $L^{2}(P Q)=L^{2}(Q P)$.

Proof. If the quadrilateral cocommutes, then $L^{2}(P Q)=L^{2}(M)=L^{2}(Q P)$. Conversely, if $L^{2}(P Q)=L^{2}(Q P)$, then $e_{P Q}=e_{Q P}$. By Theorem 3.10, $e_{P Q}$ is a scalar multiple of $e_{P} \circ e_{Q}$, so $e_{P Q} \circ e_{P Q}$ is a scalar multiple of $\left(e_{P} \circ e_{Q}\right) \circ\left(e_{P} \circ e_{Q}\right)=$ $e_{P} \circ\left(e_{Q} \circ e_{P}\right) \circ e_{Q}=e_{P} \circ\left(e_{P} \circ e_{Q}\right) \circ e_{Q}=\left(e_{P} \circ e_{P}\right) \circ\left(e_{Q} \circ e_{Q}\right)$, which is a scalar multiple of $e_{P} \circ e_{Q}$. This implies that $e_{P Q}$ is a biprojection. The corresponding subfactor has to contain both $P$ and $Q$, so is all of $M$. So $L^{2}(P Q)=L^{2}(M)$ and 
the quadrilateral cocommutes.

In fact one doesn't need the Hilbert space completion for this:

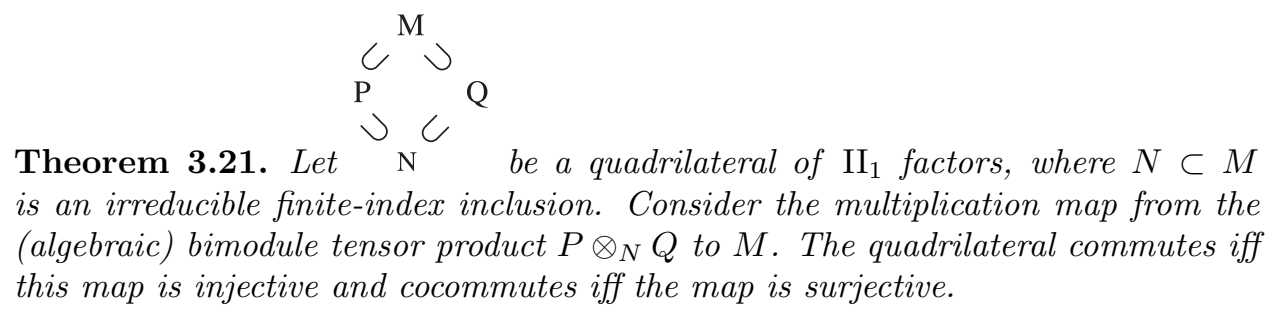

Proof. (a) Injectivity. If the algebraic map from $P \otimes_{N} Q$ to $M$ has a kernel, then it is obvious that the $L^{2}$ map does. On the other hand, the kernel $\mathfrak{K}$ of the $L^{2}$ map $\mu$ is a closed $N-N$ sub-bimodule of $L^{2}\left(M_{1}\right)$ (under the isomorphism of $L^{2}(M) \otimes_{N} L^{2}(M)$ with $\left.L^{2}\left(M_{1}\right)\right)$, and by the form of elements in the first relative commutant the orthogonal projection onto $\mathfrak{K}$ sends $M_{1}$ to itself, so there are elements of $M_{1}$ in ker $\mu$. Moreover since $M_{1} \cong M \otimes_{N} M$ the map $E_{P} \otimes E_{Q}$ produces an element of ker $\mu$ in $P e_{N} Q$.

(b) Surjectivity. The algebraic map is surjective iff $P Q=M$. Clearly $P Q=$ $M$ implies $L^{2}(P Q)=L^{2}(M)$. Conversely if $L^{2}(P Q)=L^{2}(M)$, then $e_{P Q}$ is the identity, so $M=P Q$ by Corollary 3.11.

Remark 3.22. Sano and Watatani have already shown that the quadrilateral is a cocommuting square iff $P Q=M$ under the additional hypothesis that the quadrilateral is a commuting square [33].

\section{No EXTRA STRUCTURE}

4.1. Definition. Let $N \subset M$ be an inclusion of $\mathrm{II}_{1}$ factors with associated tower $M_{-1} \subset M_{0} \subset M_{1} \subset \ldots$, where $M_{-1}=N, M_{0}=M$, and $M_{k+1}, k \geq 0$ is the von Neumann algebra on $L^{2}\left(M_{k}\right)$ generated by $M_{k}$ and $e_{k+1}$, the projection onto $L^{2}\left(M_{k-1}\right)$. Each $e_{k}$ commutes with $N$, so $\left\{1, e_{1}, \ldots, e_{k}\right\}$ generates a ${ }^{*}$-subalgebra, which we will call $T L_{k+1}$, of the $k^{\text {th }}$ relative commutant $N^{\prime} \cap M_{k}$.

To motivate the following definition (which first occurs in 23]) consider the case where $N=R^{G}, M=R^{H}$, where $G$ is a finite group of outer automorphisms of the $\mathrm{II}_{1}$ factor $R$. It is well known that, as a vector space, $N^{\prime} \cap M_{k}$ is the set of $G$-invariant functions on $X^{k+1}$, where $X=G / H$. Thus the transivity of the action of $G$ on $X$ is measured by the dimension of $N^{\prime} \cap M_{k}$ (an action is $(k+1)$-transitive if its dimension is the same as that for the full symmetric group $S_{X}$ ). Moreover any function invariant under $S_{X}$ is necessarily invariant under $G$, so the relative commutants for $R^{G} \subseteq R^{H}$ always contain a copy of those coming from $S_{X}$. The invariants under $S_{X}$ in this context are sometimes called the partition algebra, so transitivity (or rather lack of it) is measured by how much bigger $N^{\prime} \cap M_{k}$ is than the partition algebra. Now for a general subfactor $N \subseteq M$ a similar situation occurs: $N^{\prime} \cap M_{k}$ aways contains $T L_{k+1}$. Since this is, for $k>3$, strictly smaller in dimension than the partition algebra, we see that if we think of subfactors as "quantum" spaces $G / H$ they might be "more transitive" than finite group actions.

Definition 4.1. Call a finite-index subfactor $N \subseteq M k$-supertransitive (for $k>1$ ) if $N^{\prime} \cap M_{k-1}=T L_{k}$. We will say that $N \subseteq M$ is supertransitive if it is $k$ supertransitive for all $k$. 
Since $\operatorname{dim} T L_{k}$ is the same as the partition algebra for $k=1,2,3$, it is natural to call a 1,2 or 3-supertransitive subfactor transitive, 2-transitive or 3 -transitive, respectively.

Remark 4.2. $N \subseteq M$ is transitive iff it is irreducible, i.e. $N^{\prime} \cap M \cong \mathbb{C}$, it is 2-transitive iff the $N-N$ bimodule $L^{2}(M)$ has two irreducible components and 3-transitive iff $\operatorname{dim} N^{\prime} \cap M_{2} \leq 5$. Supertransitivity of $N \subseteq M$ is the same as saying its principal graph is $A_{n}$ for some $n=2,3,4, \ldots, \infty$.

Lemma 4.3. Suppose $N \subset M$ is supertransitive. If $[M: N] \geq 4$, then there is a sequence of irreducible $N-N$ bimodules $V_{0}, V_{1}, V_{2}, \ldots$ such that $L^{2}(N) \cong V_{0}$, $L^{2}(M) \cong V_{0} \oplus V_{1}$, and $V_{i} \otimes V_{j} \cong \bigoplus_{k=|i-j|}^{i+j} V_{k}$. If $[M: N]=4 \cos ^{2}\left(\frac{\pi}{n}\right)$, then the sequence terminates at $V_{l}$, where $l=\left[\frac{n-2}{2}\right]$, and the fusion rule is

$$
V_{i} \otimes V_{j} \cong \bigoplus_{k=|i-j|}^{\left(\frac{n-2}{2}\right)-\left|\left(\frac{n-2}{2}\right)-(i+j)\right|} V_{k}
$$

(see [5]).

In either case, we have

$$
\operatorname{dim}_{N} V_{k}=[M: N]^{k} T_{2 k+1}\left(\frac{1}{[M: N]}\right),
$$

where $\left\{T_{k}(x)\right\}$ is the sequence of polynomials defined recursively by $T_{0}(x)=0$, $p_{1}(x)=1$, and $T_{k+2}(x)=T_{k+1}(x)-x T_{k}(x)$.

Remark 4.4. $\operatorname{dim}_{N} V_{1}=[M: N]-1$ and $\operatorname{dim}_{N} V_{2}=[M: N]^{2}-3[M: N]+1$.

Remark 4.5. If $N \subset M$ is $2 k$-supertransitive, then there is a sequence of irreducible bimodules $V_{0}, \ldots, V_{k}$ for which the above fusion rules and dimension formula hold as long as $i+j \leq k$.

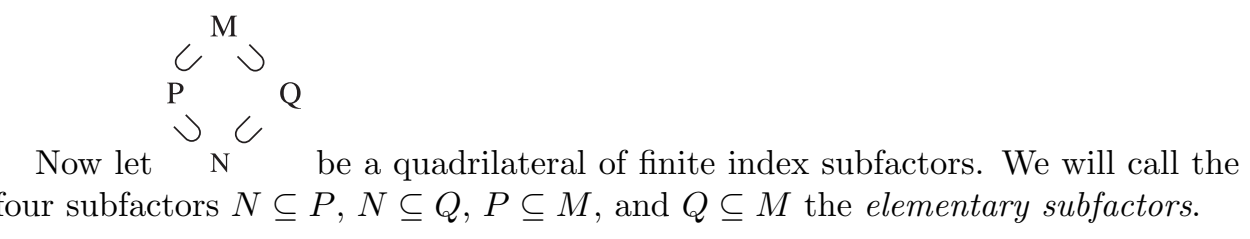

Definition 4.6. A quadrilateral as above will be said to have no extra structure if all the elementary subfactors are supertransitive.

Note that if a quadrilateral has no extra structure, the dual quadrilateral also has no extra structure.

Example 4.7. Let $G=S_{3}$ and let $H$ and $K$ be distinct two-element subgroups of $G$. Given an outer action of $G$ on a $\mathrm{II}_{1}$ factor $M$, let $N=M^{G}$, and let $P=M^{H}$ and $Q=M^{K}$. Then $N \subset P, Q \subset M$ is a quadrilateral which cocommutes (since $M^{\prime} \cap M_{2} \cong l^{\infty}(G)$ is Abelian) but does not commute (since $H K \neq K H$ ).

This quadrilateral has no extra structure since the permutation actions of $S_{2}$ and $S_{3}$ are as transitive as possible. 
4.2. Consequences of supertransitivity. Let $\overbrace{\mathrm{N}}$ be a quadrilateral of $\mathrm{II}_{1}$ factors, where $N \subset M$ is an irreducible inclusion with finite index. We also have the dual quadrilateral ${ }_{\mathrm{M}}$. Let $N \subset P \subset P_{1} \ldots$ be the tower for $N \subset P$, and similarly for $Q$.

Lemma 4.8. If $N \subseteq P$ and $N \subseteq Q$ are 2-transitive and the quadrilateral does not commute, then $L^{2}(P) \cong L^{2}(Q)$ as $N-N$ bimodules, and therefore $[P: N]=$ $[Q: N]$.

Proof. By Remark 4.2 write $L^{2}(P)=L^{2}(N) \oplus V$, where $V$ is an irreducible $N-N$ bimodule. Similarly $L^{2}(Q)=L^{2}(N) \oplus W$, for some irreducible $N-N$ bimodule $W$. Since $e_{P} e_{Q}$ is an $N-N$ intertwiner of $L^{2}(M)$ which fixes $L^{2}(N)$, leaves $L^{2}(N)^{\perp}$ invariant and whose range is contained in $L^{2}(P)$, it maps $W$ into $V$. Since $W$ is irreducible, $\operatorname{ker}\left(\left.e_{P} e_{Q}\right|_{W}\right)$ must either be zero or all of $W$. The former is impossible since that would imply $e_{P} e_{Q}=e_{N}$, which is contrary to our assumption that the quadrilateral does not commute. Thus $V \cong W$, and $\operatorname{dim}_{N} V=\operatorname{dim}_{N} W$.

Corollary 4.9. $L^{2}(P) \otimes_{N} L^{2}(Q) \cong L^{2}(P) \otimes_{N} L^{2}(P) \cong L^{2}\left(P_{1}\right)$.

Lemma 4.10. If $P \subseteq M$ is 2-transitive, then $L^{2}(P Q P)=L^{2}(M)$.

Proof. By Remark 4.2 write $L^{2}(M) \cong L^{2}(P) \oplus W$ for some irreducible $P-P$ bimodule $W$. Since $L^{2}(P Q P)$ is a $P-P$ submodule of $L^{2}(M)$ which is strictly larger than $L^{2}(P)$, it must in fact be equal to $L^{2}(M)$.

Remark 4.11. Suppose all of the elementary inclusions of the quadrilateral are $2 k$ supertransitive for some $k \geq 1$. Then the elementary inclusions of the dual quadrilateral are also $2 k$-supertransitive. Putting together Remark 3.3. Lemma 4.10, and Lemma 4.8, we find that as an $N-N$ bimodule, $L^{2}(M)$, is a quotient of $\otimes_{N}^{3} L^{2}(P)$. If $k \geq 3$, then the irreducible submodules of $L^{2}(M)$ belong to $\left\{V_{0}, V_{1}, V_{2}, V_{3}\right\}$, where the $\left\{V_{i}\right\}$ are as in Remark 4.5 for the 6-supertransitive inclusion $N \subset P$. Similarly, as an $M-M$ bimodule, $L^{2}\left(M_{1}\right)$ is a quotient of $\otimes_{M}^{3} L^{2}(\bar{P})$. We will write $U_{0}, U_{1}$, etc., for the irreducible $M-M$ bimodules occurring in the decomposition of the first $k$ tensor powers of $L^{2}(\bar{P})$.

For convenience we state the following rewording of a lemma in [30, which we will be using repeatedly:

Lemma 4.12. If the $N-N$ bimodule decomposition of $L^{2}(M)$ contains $k$ copies of the $N-N$ bimodule $R$, then $k \leq \operatorname{dim}_{N} R$. In particular, $L^{2}(M)$ contains only one copy of $L^{2}(N)$.

Proof. ${ }_{N} L^{2}(M)_{N} \cong\left({ }_{N} L^{2}(M)_{M}\right) \otimes_{M}\left({ }_{M} L^{2}(M)_{N}\right)$, so if ${ }_{N} L^{2}(M)_{N}$ contains $k$ copies of $R$, then by Frobenius reciprocity, $R \otimes_{N}\left({ }_{N} L^{2}(M)_{M}\right)$ contains $k$ copies of the $N-M$ bimodule ${ }_{N} L^{2}(M)_{M}$, which implies that

$$
\operatorname{dim}_{N}\left(R \otimes_{N} L^{2}(M)_{M}\right)=\operatorname{dim}_{N}(R)[M: N] \geq k \cdot \operatorname{dim}_{N}\left({ }_{N} L^{2}(M)_{M}\right)=k[M: N] .
$$


Lemma 4.13. If $N \subseteq P$ and $N \subseteq Q$ are 4-supertransitive and the quadrilateral does not commute, then the $N-N$ bimodule $L^{2}(P Q)$ is isomorphic to one of the following: $V_{0} \oplus 2 V_{1} \oplus V_{2}, V_{0} \oplus 3 V_{1} \oplus V_{2}$, or $V_{0} \oplus 3 V_{1}$, where the $V_{i}$ are as in Lemma 4.3 (for the 4-supertransitive inclusion $N \subset P$ ).

Proof. By Corollary 3.2, $L^{2}(P Q)$ is isomorphic to a submodule of $L^{2}\left(P_{1}\right)$. A decomposition of $L^{2}\left(P_{1}\right)$ into $N-N$ submodules corresponds to a decomposition of the identity in $N^{\prime} \cap P_{3}$.

If $\operatorname{dim}\left(N^{\prime} \cap P_{3}\right)=14$, then $N^{\prime} \cap P_{3} \cong M_{2}(\mathbb{C}) \oplus M_{3}(\mathbb{C}) \oplus \mathbb{C}$, where the first summand corresponds to $V_{0}$, the second to $V_{1}$, and the third to $V_{2}$. So $L^{2}\left(P_{1}\right) \cong 2 V_{0} \oplus 3 V_{1} \oplus V_{2}$. By Lemma 4.12, $L^{2}(P Q)$ contains only one copy of $L^{2}(N)$. Also, by Lemma 4.8, $L^{2}(Q) \cong L^{2}(P)$, but $L^{2}(P) \neq L^{2}(Q)$, so $L^{2}(P Q)$ contains at least two copies of $V_{1}$. It is impossible that $L^{2}(P Q) \cong V_{0} \oplus 2 V_{1}$, since that would imply that $L^{2}(P Q)=L^{2}(P+Q)=L^{2}(Q P)=L^{2}(M)$, which would imply that

$$
[M: P]=\frac{\operatorname{dim}_{N} L^{2}(M)}{\operatorname{dim}_{N} L^{2}(P)}<2 .
$$

That leaves the three possibilities above. If $\operatorname{dim}\left(N^{\prime} \cap P_{3}\right)<14$, then the argument is essentially the same, except there is no $V_{2}$, so only one possibility remains.

\subsection{Cocommuting quadrilaterals with no extra structure.}

Notation. From now on the supertransitivity hypotheses will guarantee that $[M: P]=[M: Q]$. We introduce the following notational conventions:

$$
[M: P]=\beta, \quad[P: N]=\alpha, \quad[M: N]=\gamma=1 / \tau,
$$

which we will use without further mention.

Lemma 4.14. If $N \subset P$ and $N \subset Q$ are 2-transitive, then $e_{P} e_{Q} e_{P}=e_{N}+$ $\lambda\left(e_{P}-e_{N}\right)$, where

$$
\lambda=\frac{\operatorname{tr}\left(e_{\bar{P}} \bar{Q}\right)^{-1}-1}{[P: N]-1} .
$$

Proof. That $e_{P} e_{Q} e_{P}=e_{N}+\lambda\left(e_{P}-e_{N}\right)$ for some $\lambda$ follows from the fact that $e_{P} e_{Q} e_{P}$ is an $N-N$ intertwiner of $L^{2}(P) \cong V_{0} \oplus V_{1}$, which is the identity on $L^{2}(N)$. To compute $\lambda$, note that $\operatorname{tr}\left(e_{P} e_{Q} e_{P}\right)=\operatorname{tr}\left(e_{N}\right)+\lambda \operatorname{tr}\left(e_{P}-e_{N}\right)=\frac{1}{\gamma}+\lambda \frac{\alpha-1}{\gamma}$. Solving for $\lambda$ and using $\operatorname{tr}\left(e_{P} e_{Q} e_{P}\right)=\frac{1}{\gamma \operatorname{tr}\left(e_{\bar{P} \bar{Q}}\right)}$ (by Lemma 3.16) completes the proof.

Corollary 4.15. $\operatorname{tr}\left(\left(e_{P} e_{Q} e_{P}\right)^{2}\right)=\frac{1+\lambda^{2}([P: N]-1)}{[M: N]}$.

Lemma 4.16. If the quadrilateral cocommutes and $e_{\bar{P} \bar{Q}} e_{\bar{Q} \bar{P}}=e_{\bar{Q} \bar{P}} e_{\bar{P} \bar{Q}}$, then

$$
\begin{aligned}
& \operatorname{dim}_{M} L^{2}(\bar{P} \bar{Q}+\bar{Q} \bar{P}) \\
& \quad=[M: P]^{2}\left(2-\frac{[M: P]}{[P: N]}\left(1+\left(\frac{[P: N]-[M: P]}{[M: N]-[M: P]}\right)^{2}([P: N]-1)\right)\right) .
\end{aligned}
$$

Proof. Since $e_{\bar{P}} e_{\bar{Q}} e_{\bar{Q} \bar{P}}=e_{\bar{Q} \bar{P}} e_{\bar{P} \bar{Q}}, \operatorname{dim}_{M} L^{2}(\bar{P} \bar{Q}+\bar{Q} \bar{P})=\gamma\left(2 \operatorname{tr}\left(e_{\bar{P} \bar{Q}}\right)-\operatorname{tr}\left(e_{\bar{P}} \bar{Q}_{\bar{Q}} e_{\bar{Q} \bar{P}}\right)\right)$.

Since the quadrilateral cocommutes,

$$
\operatorname{tr}\left(e_{\bar{P} \bar{Q}}\right)=\frac{\operatorname{dim}_{M} L^{2}(\bar{P} \bar{Q})}{\gamma}=\frac{\operatorname{dim}_{M} L^{2}(\bar{P}) \operatorname{dim}_{M} L^{2}(\bar{Q})}{\gamma}=\frac{\beta^{2}}{\gamma}=\frac{\beta}{\alpha} .
$$


By (the dual version of) Lemma 3.17. $\operatorname{tr}\left(e_{\bar{P}} e_{\bar{Q}} e_{\bar{P}}\right)=\left(\delta \operatorname{tr}\left(e_{\bar{P} \bar{Q}}\right)\right)^{2} \operatorname{tr}\left(\left(e_{P} e_{Q} e_{P}\right)^{2}\right)$ $=\operatorname{tr}\left(e_{\bar{P} \bar{Q}}\right)^{2}\left(1+\lambda^{2}(\alpha-1)\right)=\left(\frac{\beta}{\alpha}\right)^{2}\left(1+\lambda^{2}(\alpha-1)\right)$. Also, since $\operatorname{tr}\left(e_{\bar{P} \bar{Q}}\right)=\frac{\beta}{\alpha}$, we have $\lambda=\frac{\alpha-\beta}{\gamma-\beta}$. Putting all this together gives the result.

Corollary 4.17. In the special case that $[M: P]=[P: N]-1$, the formula becomes $\operatorname{dim}_{M} L^{2}(\bar{P} \bar{Q}+\bar{Q} \bar{P})=[M: P]^{2}+[M: P]-1$.

Theorem 4.18. If the quadrilateral cocommutes but does not commute, and $N \subseteq P$ and $N \subseteq Q$ are 4-supertransitive, then $N$ is the fixed point algebra of an outer $S_{3}$ action on $M$.

Proof. Since the quadrilateral does not commute, $L^{2}(P) \cong L^{2}(Q)$ as $N-N$ bimodules, by Lemma 4.8. Since the quadrilateral cocommutes, $L^{2}(M)=L^{2}(P Q)$, and since $N^{\prime} \cap P_{3} \leq 14$, by Lemma 4.13 the isomorphism type of $L^{2}(M)$ is one of $V_{0} \oplus 2 V_{1} \oplus V_{2}, V_{0} \oplus 3 V_{1} \oplus V_{2}$, or $V_{0} \oplus 3 V_{1}$. For each of these cases we can explicitly compute $\beta$ as a function of $\alpha$ using the formula $\beta=\gamma / \alpha=\operatorname{dim}_{N} L^{2}(M) / \alpha$ and the dimension formulas of Remark 4.4 .

Case 1: $L^{2}(M) \cong V_{0} \oplus 3 V_{1} \oplus V_{2}$

In this case,

$$
[\bar{P}: M]=\beta=\frac{\operatorname{dim}_{N} L^{2}(M)}{\alpha}=\frac{1+3(\alpha-1)+\alpha^{2}-3 \alpha+1}{\alpha}=\alpha-\frac{1}{\alpha} .
$$

Since the quadrilateral cocommutes, by Corollary 3.19 we have

$$
\operatorname{dim}_{M} L^{2}(\bar{P} \bar{Q})=\left(\alpha-\frac{1}{\alpha}\right)^{2}
$$

But then the dimension of its orthogonal complement (in $\left.L^{2}\left(M_{1}\right)\right)$ is

$$
\operatorname{dim}_{M} L^{2}\left(M_{1}\right)-\operatorname{dim}_{M} L^{2}(\bar{P} \bar{Q})=\alpha^{2}-1-\left(\alpha-\frac{1}{\alpha}\right)^{2}=1-\frac{1}{\alpha^{2}}<1,
$$

which is impossible by Lemma 4.12 .

Case 2: $L^{2}(M) \cong V_{0} \oplus 3 V_{1}$

In this case,

$$
\beta=\frac{1+3(\alpha-1)}{\alpha}=3-\frac{2}{\alpha},
$$

which necessarily equals $4 \cos ^{2} \frac{\pi}{5}$. (The only other admissible index value less than three is two, but that would imply that the total index is four and then the quadrilateral would commute.) Then we have the identity $\beta^{2}=3 \beta-1$, and $\alpha=2 \beta$. Since $L^{2}(M) \cong V_{0} \oplus 3 V_{1}$, any intermediate subfactor must have index equal to

$$
\frac{1+3(\alpha-1)}{1+k(\alpha-1)}
$$

for $k=1$ or $k=2$. So to eliminate this case it suffices to find a proper subfactor of $M$ with an integer-valued index, for which it suffices to find an $M-M$ submodule of $L^{2}\left(M_{1}\right)$ whose dimension over $M$ is 1 .

$L^{2}(\bar{P}+\bar{Q})$ has $M$-dimension $2 \operatorname{dim}_{M} L^{2}(\bar{P})-\operatorname{dim}_{M} L^{2}(M)=2 \beta-1$. Its orthogonal complement in $L^{2}(\bar{P} \bar{Q})$, which we shall call $T$, has $M$-dimension $\operatorname{dim}_{M} L^{2}(\bar{P} \bar{Q})-$ $\operatorname{dim}_{M} L^{2}(\bar{P}+\bar{Q})=\beta^{2}-(2 \beta-1)=\beta$. Since $\beta<3$, if $T$ is reducible, one of its irreducible components must have $M$-dimension 1 , and we are finished. Similarly, if $T^{\prime}$, the orthogonal complement of $L^{2}(\bar{P}+\bar{Q})$ in $L^{2}(\bar{Q} \bar{P})$, is reducible, then we get a submodule of $M$-dimension 1 . 
If $T$ and $T^{\prime}$ are both irreducible, then $L^{2}(\bar{P} \bar{Q}) \cap L^{2}(\bar{Q} \bar{P})=L^{2}(\bar{P}+\bar{Q})$. Then if $S$ is the orthogonal complement of $L^{2}(\bar{P} \bar{Q}+\bar{Q} \bar{P})$ in $L^{2}\left(M_{1}\right)$, we have

$$
\begin{aligned}
\operatorname{dim}_{M} S & =\operatorname{dim}_{M} L^{2}\left(M_{1}\right)-\left(2 \operatorname{dim}_{M} L^{2}(\bar{P} \bar{Q})-\operatorname{dim}_{M} L^{2}(\bar{P}+\bar{Q})\right) \\
& =2 \beta^{2}-\left(2 \beta^{2}-(2 \beta-1)\right)=2 \beta-1 .
\end{aligned}
$$

Since $\operatorname{dim}\left(M^{\prime} \cap M_{2}\right)=\operatorname{dim}\left(N^{\prime} \cap M_{1}\right)=10, S$ must break into 3 components, one of which must have $M$-dimension 1 .

Case 3: $L^{2}(M) \cong V_{0} \oplus 2 V_{1} \oplus V_{2}$

In this case

$$
\beta=\frac{1+2(\alpha-1)+\alpha^{2}-3 \alpha+1}{\alpha}=\alpha-1 .
$$

Note that $\operatorname{dim}\left(N^{\prime} \cap M_{1}\right)=6$, and therefore also $\operatorname{dim}\left(M^{\prime} \cap M_{2}\right)=6$. Because $L^{2}(M) \subset L^{2}(\bar{P}) \subset L^{2}(\bar{P}+\bar{Q}) \subset L^{2}(\bar{P} \bar{Q}) \subset L^{2}\left(M_{1}\right)$ is a strictly increasing chain of $M-M$ bimodules ( $\bar{P} \bar{Q}$ cannot be all of $M_{1}$ because the quadrilateral does not commute), $M^{\prime} \cap M_{2}$ must be Abelian. If we let $x=\beta$ (so that $\alpha=x+1$ ), then $\gamma=x^{2}+x$, and by Corollary 4.17 we have that $\operatorname{dim}_{M} L^{2}(\bar{P} \bar{Q}+\bar{Q} \bar{P})=x^{2}+x-1$, and so the dimension of its orthogonal complement in $L^{2}\left(M_{1}\right)$ is 1 .

It is then easy to see that the dimensions of the six distinct irreducible submodules of $L^{2}\left(M_{1}\right)$ are $1, x-1, x-1, x^{2}-2 x-1, x^{2}-2 x-1,1$. But then summing we find that $2 x^{2}-2 x-2=\operatorname{dim}_{M} L^{2}\left(M_{1}\right)=x^{2}+x$, which implies that $x=2$. So $[\bar{P}: M]=[\bar{Q}: M]=2$, and $\left[M_{1}: M\right]=6$.

So by Goldman's theorem [11, $M_{1}$ is the crossed product of $M$ by $S_{3}$, or, equivalently, $N$ is the fixed point subalgebra of an outer $S_{3}$ action on $M$.

\section{RESTRICTIONS ON THE PRINCIPAL GRAPH}

If the quadrilateral has no extra structure, then we obtain severe restrictions on the principal graph. Specifically, for a noncommuting, noncocommuting quadrilateral with no extra structure the principal graph is completely determined.

\subsection{Structural restrictions.}

Lemma 5.1. If the quadrilateral neither commutes nor cocommutes, and all the elementary subfactors are 6-supertransitive, then $N^{\prime} \cap M_{1}$ and $M^{\prime} \cap M_{2}$ both have more than two simple summands.

Proof. First suppose that $N^{\prime} \cap M_{1}$ and $M^{\prime} \cap M_{2}$ both have exactly two simple summands. Then $L^{2}(M)=V_{0} \oplus k V_{1}$ for some integer $k$. So we have

$$
\beta=\frac{\gamma}{\alpha}=\frac{\operatorname{dim}_{N}\left(V_{0} \oplus k V_{1}\right)}{\alpha}=\frac{1+k(\alpha-1)}{\alpha}=k-\frac{k-1}{\alpha}<k .
$$

By Lemma 4.12, $k \leq \operatorname{dim}_{N} V=\alpha-1<\alpha$, and so $\beta<\alpha$. But we can perform the same calculation in the dual quadrilateral to find that $\alpha<\beta$, which is a contradiction.

Now suppose that only $M^{\prime} \cap M_{2}$ has exactly two simple summands, and write $L^{2}\left(M_{1}\right) \cong U_{0} \oplus l U_{1}$. Note that because of the 6 -supertransitivity hypothesis, the first few tensor powers of $U_{1}$ decompose according to the fusion rules of Lemma 4.3. By Lemma 4.13. $L^{2}(\bar{P} \bar{Q}) \cong U_{0} \oplus 3 U_{1}$, and since the quadrilateral does not commute, by Corollary $3.20, L^{2}(\bar{P} \bar{Q}) \neq L^{2}(\bar{Q} \bar{P})$, so $l$ must be at least 4 . By Lemma 4.10 and Remark 3.3 , $L^{2}\left(M_{1}\right)$ is a quotient of $L^{2}(\bar{P} \bar{Q}) \otimes_{M} L^{2}(\bar{P}) \cong\left(U_{0} \oplus 3 U_{1}\right) \otimes_{M}$ 
$\left(U_{0} \oplus U_{1}\right) \cong 4 U_{0} \oplus 7 U_{1} \oplus 3 U_{2}$, where the last isomorphism comes from the fusion rule $U_{1} \otimes_{M} U_{1} \cong U_{0} \oplus U_{1} \oplus U_{2}$ (if $\alpha<3$, then $U_{2}=0$ ). So we find that $4 \leq l \leq 7$.

Similarly, $L^{2}(M)$ is a quotient of $L^{2}(P Q) \otimes_{N} L^{2}(P)$, which in all cases of Lemma 4.13 is a quotient of $\left(V_{0} \oplus 3 V_{1} \oplus V_{2}\right) \otimes_{N}\left(V_{0} \oplus V_{1}\right) \cong V_{0} \oplus 8 V_{1} \oplus 5 V_{2} \oplus V_{3}$. Thus we may write $L^{2}(M) \cong V_{0} \oplus a V_{1} \oplus b V_{2} \oplus c V_{3}$, where $a, b$, and $c$ are integers such that $2 \leq a \leq 8,0 \leq b \leq 5$, and $0 \leq c \leq 1$, and $b$ and $c$ are not both 0 .

But because we have $\operatorname{dim}\left(N^{\prime} \cap M_{1}\right)=\operatorname{dim}\left(M^{\prime} \cap M_{2}\right)$, we necessarily have $a^{2}+$ $b^{2}+c^{2}=l^{2}$. A quick examination reveals that the only possibility is that $l=5$, $c=0$, and $\{a, b\}=\{3,4\}$. But if $l=5$, then

$$
\alpha=\frac{\gamma}{\beta}=\frac{\left[M_{1}: M\right]}{[\bar{P}: M]}=5-\frac{4}{\beta}<5,
$$

which implies that $a \leq \operatorname{dim}_{N} V_{1}<4$ (by Lemma 4.12), so we may assume that $a=3$ and $b=4$. Then

$$
\beta=\frac{\operatorname{dim}_{N} V_{0} \oplus 3 V_{1} \oplus 4 V_{2}}{\alpha}=\frac{1+3(\alpha-1)+4\left(\alpha^{2}-3 \alpha+1\right)}{\alpha}=4 \alpha^{2}-9 \alpha+2,
$$

and since $\alpha \geq 3$, we must have $\beta \geq 4$, and then also $\alpha=5-4 / \beta \geq 4$, so the generic fusion rules of Lemma 4.3 apply.

Then as an $N-N$ bimodule, $L^{2}\left(M_{1}\right) \cong L^{2}(M) \otimes_{N} L^{2}(M) \cong\left(V_{0} \oplus 3 V_{1} \oplus 4 V_{2}\right)$ $\otimes_{N}\left(V_{0} \oplus 3 V_{1} \oplus 4 V_{2}\right) \cong 10 V_{0} \oplus 39 V_{1} \oplus 41 V_{2} \oplus 12 V_{3} \oplus 16\left(V_{2} \otimes_{N} V_{2}\right)$, where the last isomorphism comes from the fusion rules $V_{1} \otimes_{N} V_{1} \cong V_{0} \oplus V_{1} \oplus V_{2}$ and $V_{1} \otimes_{N} V_{2} \cong$ $V_{1} \oplus V_{2} \oplus V_{3}$. Since the $N-N$ intertwiner space of $L^{2}\left(M_{1}\right)$ is $N^{\prime} \cap M_{3}$, this implies that $\operatorname{dim}\left(N^{\prime} \cap M_{3}\right) \geq 10^{2}+39^{2}+41^{2}+12^{2}=3446$.

On the other hand, as an $M-M$ bimodule, $L^{2}\left(M_{2}\right) \cong L^{2}\left(M_{1}\right) \otimes_{M} L^{2}\left(M_{1}\right)$ $\cong L^{2}(M) \oplus 5 U_{1} \otimes L^{2}(M) \oplus 5 U_{1} \cong 26 U_{0} \oplus 35 U_{1} \oplus 25 U_{2}$, so $\operatorname{dim}\left(M^{\prime} \cap M_{4}\right)=26^{2}+35^{2}+$ $25^{2}=2526$. But this contradicts the fact that $\operatorname{dim}\left(N^{\prime} \cap M_{3}\right)=\operatorname{dim}\left(M^{\prime} \cap M_{4}\right)$.

Lemma 5.2. If the quadrilateral neither commutes nor cocommutes and all the elementary subfactors are 6-supertransitive, then $[N: P]$ and $[M: P]$ are both less than 4.

Proof. Suppose on the contrary that the hypotheses are satisfied and that $\alpha \geq 4$. (There is no loss of generality here since if only $\beta \geq 4$ we may consider the dual quadrilateral instead.) Then by Lemma 5.1. $N^{\prime} \cap M_{1}$ has at least three simple summands. Because the quadrilateral is not cocommuting, by Corollary 3.20 . $L^{2}(P Q) \neq L^{2}(Q P)$, but they must have the same dimension since by Corollary 3.15, $\operatorname{tr}\left(e_{P Q}\right)=\operatorname{tr}\left(e_{Q P}\right)$. We consider three cases, corresponding to the three cases of Lemma 4.13 .

Case 1: $L^{2}(P Q) \cong V_{0} \oplus 3 V_{1}$. Then also $L^{2}(Q P) \cong V_{0} \oplus 3 V_{1}$. Note that these two bimodules intersect in $L^{2}(P+Q) \cong V_{0} \oplus 2 V_{1}$, so $L^{2}(P Q+Q P) \cong V_{0} \oplus 4 V_{1}$. Since $N^{\prime} \cap M_{1}$ has a third summand, $L^{2}(M)$ must also contain an irreducible submodule whose dimension is at least as great as that of $V_{2}$, by Lemma 4.3. so we find that $\gamma=\operatorname{dim}_{N} L^{2}(M) \geq \operatorname{dim}_{N} V_{0} \oplus 4 V_{1} \oplus V_{2} \geq 1+4(\alpha-1)+\left(\alpha^{2}-3 \alpha+1\right)=\alpha^{2}+\alpha-2$, and so $\beta=\gamma / \alpha=\alpha+1-2 / \alpha>\alpha$.

Case 2: $L^{2}(P Q) \cong V_{0} \oplus 2 V_{1} \oplus V_{2}$. Then $L^{2}(P Q+Q P) \cong V_{0} \oplus 2 V_{1} \oplus 2 V_{2}$. So $\gamma \geq \operatorname{dim}_{N} V_{0} \oplus 2 V_{1} \oplus 2 V_{2}=2 \alpha^{2}-4 \alpha+1$, and again we find that $\beta=2 \alpha-4+1 / \alpha>\alpha$ (because $\alpha \geq 4$ ).

Case 3: $L^{2}(P Q) \cong V_{0} \oplus 3 V_{1} \oplus V_{2}$. Then $L^{2}(P Q+Q P)$ contains either at least four copies of $V_{1}$ or at least two copies of $V_{2}$ and again we find that $\beta>\alpha$. 
But since $\beta>\alpha \geq 4$, we can perform these same calculations in the dual quadrilateral to deduce that $\alpha>\beta$, which is absurd.

Lemma 5.3. If the quadrilateral neither commutes nor cocommutes and all the elementary subfactors are 6-supertransitive, then $[P: N]=[M: P]$.

Proof. By the previous lemma we may assume that $\alpha$ and $\beta$ are both less than four. Because $\alpha<4, \operatorname{dim}_{N} V_{1}<3$, so by Lemma 4.12, $L^{2}(M)$ contains at most, and therefore exactly, two copies of $V_{1}$, and so $L^{2}(P Q) \cong V_{0} \oplus 2 V_{1} \oplus V_{2}$. Now $L^{2}(M)$ is a quotient of $L^{2}(P Q) \otimes_{N} L^{2}(P) \cong\left(V_{0} \oplus 2 V_{1} \oplus V_{2}\right) \otimes_{N}\left(V_{0} \oplus V_{1}\right) \cong 3 V_{0} \oplus 6 V_{1} \oplus 4 V_{2} \oplus V_{3}$, so it contains at most four copies of $V_{2}$ and at most one copy of $V_{3}$ (and nothing higher). Also, since $L^{2}(Q P)$ is isomorphic, but not equal, to $L^{2}(P Q), L^{2}(M)$ contains at least two copies of $V_{2}$.

So we may write $L^{2}(M) \cong V_{0} \oplus 2 V_{1} \oplus b V_{2} \oplus c V_{3}$, with $2 \leq b \leq 4$ and $0 \leq c \leq 1$. Similarly, we may write $L^{2}\left(M_{1}\right) \cong U_{0} \oplus 2 U_{1} \oplus b^{\prime} U_{2} \oplus c^{\prime} U_{3}$, with $2 \leq b^{\prime} \leq 4$ and $0 \leq c^{\prime} \leq 1$. Since $1^{2}+2^{2}+b^{2}+c^{2}=\operatorname{dim}\left(N^{\prime} \cap M_{1}\right)=\operatorname{dim}\left(M^{\prime} \cap M_{2}\right)=1^{2}+2^{2}+b^{\prime 2}+c^{\prime 2}$ and $c$ and $c^{\prime}$ are each either 0 or 1 , we must have $b=b^{\prime}$ and $c=c^{\prime}$.

Define the function

$$
\begin{aligned}
f_{b, c}(x) & =\left[1+2(x-1)+b\left(x^{2}-3 x+1\right)+c\left(x^{3}-5 x^{2}+6 x-1\right)\right] / x \\
& =c x^{2}+(b-5 c) x+(2-3 b+6 c)+\frac{(b-c-1)}{x} .
\end{aligned}
$$

Then $f_{b, c}(\alpha)=\beta$ and $f_{b, c}(\beta)=\alpha$. Define $g_{b, c}(x)=f_{b, c}(x)-x$. Then $g_{b, c}^{\prime}(x)$ is either $b-1-(b-1) / x^{2}$, or $2 x+b-6-(b-2) / x^{2}$, depending upon whether $c$ is 0 or 1 . In either case, $g^{\prime}(x)$ is positive when $x \geq 2$ and so $g(x)$ is then an increasing function.

Now if $\alpha>\beta$, then $g_{b, c}(\beta)=f_{b, c}(\beta)-\beta=\alpha-\beta>0$, and since $\alpha>\beta$ and $g_{b, c}(x)$ is increasing, $g_{b, c}(\alpha)>0$ as well, so we also have $\beta>\alpha$, which is a contradiction. Similarly we find that $\beta>\alpha$ is impossible. Therefore we must have $\beta=\alpha$.

\subsection{The principal graph.}

Lemma 5.4. There does not exist a noncommuting quadrilateral of subfactors with $L^{2}(M) \cong V_{0} \oplus 2 V_{1} \oplus 2 V_{2}$ and with the principal graph of the elementary subfactors equal to $A_{11}$.

Proof. Suppose such a quadrilateral exists. Then $L^{2}\left(M_{1}\right) \cong L^{2}(M) \otimes_{N} L^{2}(M) \cong$ $9 V_{0} \oplus 20 V_{1} \oplus 20 V_{2} \oplus 12 V_{3} \oplus 4 V_{4}$, and $L^{2}\left(M_{2}\right) \cong L^{2}\left(M_{1}\right) \otimes_{N} L^{2}\left(M_{1}\right) \cong 89 V_{0} \oplus 222 V_{1}$ $\oplus 254 V_{2} \oplus 196 V_{3} \oplus 108 V_{4} \oplus 32 V_{5}$, by the $A_{11}$ fusion rules. (Lemma 4.3 with $n=12$ gives $V_{i} \otimes_{N} V_{j}=\bigoplus_{|i-j|}^{5-|5-(i+j)|} V_{k}$.)

Recalling the principle that each level of the Bratteli diagram for the tower of relative commutants is obtained by reflecting the previous level and adding some "new stuff", with the rule that the "new stuff" connects only to the "old new stuff" (see [12]), it is easy to deduce that the Bratteli diagram must include the graph in Figure 5.5 .

Let $m$ and $n$ be the number of bonds which connect the two " 2 " $\mathrm{s}$ in the fourth row with " 12 " in the fifth row, respectively. Then we must have $2 m+2 n=12$, or $m+n=6$. By the reflection principle, there must also be $m$ and $n$ bonds connecting " 12 " with " $x$ " and " $y$ " respectively, as well as " $x$ " and " $y$ " with " 196 ". This implies that $x \geq 20+12 m, y \geq 20+12 n$, and $196 \geq m(20+12 m)+n(20+12 n)=$ $20(m+n)+12\left(m^{2}+n^{2}\right)$, which is absurd since $m+n=6$. 


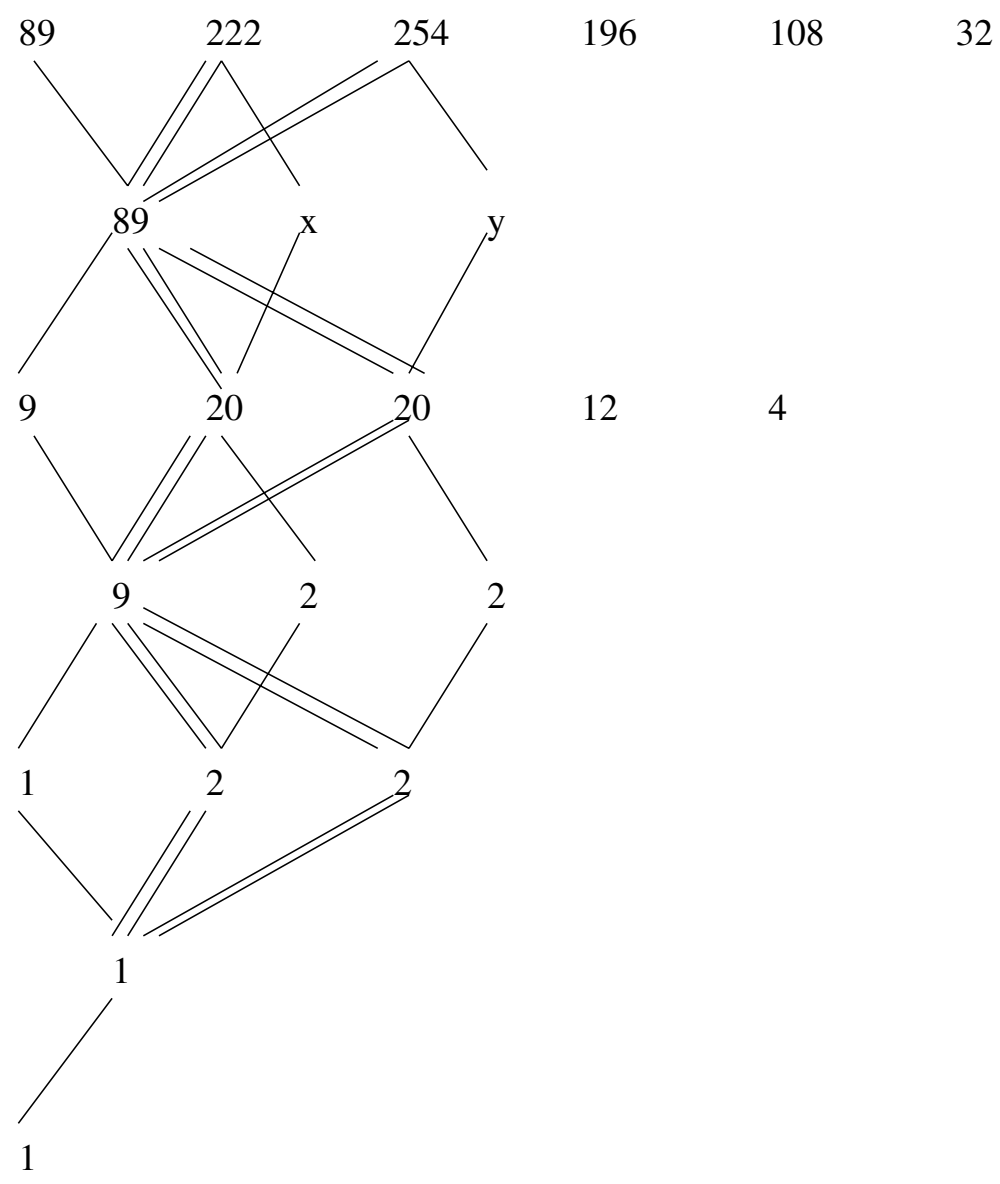

FiguRE 5.5.

Lemma 5.6. If the quadrilateral neither commutes nor cocommutes, and the elementary inclusions are 6-supertransitive, then $[P: N]=[M: P]=2+\sqrt{2}$ and $L^{2}(M) \cong V_{0} \oplus 2 V_{1} \oplus 2 V_{2} \oplus V_{3}$.

Proof. As in the proof of Lemma 5.3. there are six possible isomorphism types for $L^{2}(M) \cong V_{0} \oplus 2 V_{1} \oplus b V_{2} \oplus c V_{3}$, corresponding to $b=2,3,4$ and $c=0,1$. We will eliminate them all except $b=2, c=1$.

Let $x=\alpha$. From the proof, and the conclusion, of Lemma 5.3 we have

$$
c x^{3}+(b-5 c-1) x^{2}+(2-3 b+6 c) x+(b-c-1)=0 .
$$

Let us consider the cases one at a time:

$c=0, b=2$

Then $x=2+\sqrt{3}$ and the only principal graphs possible for $N \subseteq P$ are $A_{11}$ and $E_{6}$. But $E_{6}$ is not 4 -supertansitive and $A_{11}$ was eliminated in Lemma 5.4 $c=0, b=3$ Then $2 x^{2}-7 x+2=0$, neither root of which is an allowed index value. 
$c=0, b=4$

Then $3 x^{2}-10 x+3=0$, so $\alpha=3$, which implies $\operatorname{dim}_{N}\left(V_{2}\right)=1$, which is impossible by Lemma 4.12 .

$c=1, b=3$

Then $x^{3}-3 x-x+1=0$ or $x\left(x^{2}-3 x+1\right)=2 x-1$, which $\operatorname{implies}_{\operatorname{dim}_{N}}\left(V_{2}\right)<2$. Again by Lemma 4.12 this is impossible.

$c=1, b=4$

Then $x^{3}-2 x^{2}-4 x+2=0$. The largest root of this equation is between 3 and $4 \cos ^{2} \pi / 7$, so it is not a possible index value.

Finally, in the case $c=1, b=2, x\left(x^{2}-4 x+2\right)=0$, so $\alpha=2+\sqrt{2}$ (which is $\left.4 \cos ^{2} \pi / 8\right)$ ).

Corollary 5.7. With the hypotheses of the previous lemma, $\operatorname{tr}\left(e_{P Q}\right)=\frac{1}{\sqrt{2}}, \operatorname{tr}\left(e_{P} e_{Q}\right)$ $=\frac{1}{4+3 \sqrt{2}}$ and the angle between $P$ and $Q$ is $\cos ^{-1}(\sqrt{2}-1)$.

Proof. By 2-transitivity we know that $e_{P} e_{Q} e_{P}=e_{N}+t\left(e_{P}-e_{N}\right)$ for some number $t$ which is the square of the cosine of the angle. Moreover, by Lemma 4.13 we know that $\operatorname{dim}_{N}\left(L^{2}(P Q)\right)=1+3(1+\sqrt{2})$. Taking the trace, using Proposition 3.14 and solving for $t$ we are done.

Theorem 5.8. Let $\stackrel{\mathrm{P}}{\circlearrowright} \mathrm{C}_{\mathrm{Q}}^{\mathrm{M}}$ be a noncommuting noncocommuting quadrilateral with all elementary inclusions 6-supertransitive. Then $[M: P]=[M: Q]=[P:$ $N]=[Q: N]=2+\sqrt{2}$ and the principal and dual principal graphs for $N \subset M$ are both

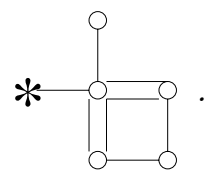

Proof. Reduction to this one case is a consequence of the previous results. We need only compute the principal graph. Since there is no subfactor with principal graph $D_{5}$, all the elementary subfactors must have principal graph $A_{7}$. Thus there are only the 4 possible isomorphism types $V_{0}, V_{1}, V_{2}$ and $V_{3}$ for the $N-N$ bimodules in $L^{2}(M), L^{2}\left(M_{1}\right), \ldots$; i.e., the Bratteli diagram for the tower of relative commutants $N^{\prime} \cap M_{K}$ has at most 4 simple summands for $k$ odd. Since there are 4 simple

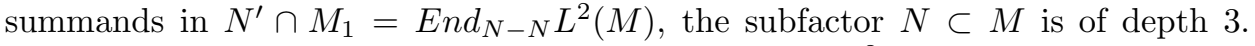
Moreover if we let $V_{a}=V_{0} \oplus V_{3}$ and $V_{b}=V_{1} \oplus V_{2}$, then $L^{2}(M) \cong V_{a} \oplus 2 V_{b}$, and the fusion rules are very simple: $V_{a} \otimes V_{a}=2 V_{a}, V_{a} \otimes V_{b}=2 V_{b}$, and $V_{b} \otimes V_{b}=2 V_{A} \oplus 4 V_{b}$. So $L^{2}\left(M_{1}\right) \cong L^{2}(M) \otimes L^{2}(M) \cong 10 V_{a} \oplus 24 V_{b} \cong 10 V_{0} \oplus 24 V_{1} \oplus 24 V_{2} \oplus 10 V_{3}$, and there is only one way to fill in the $N^{\prime} \cap M_{2}$ level of the Bratteli diagram for the tower of relative commutants, which will thus begin as in Figure 5.9. 


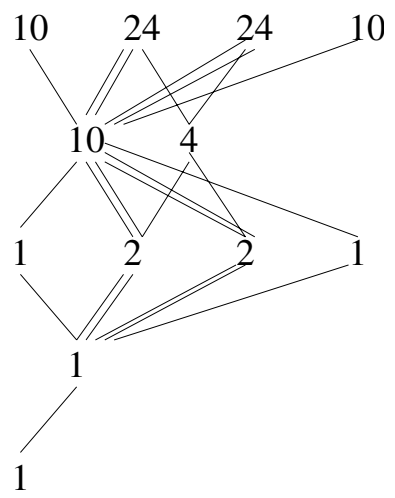

FiguRE 5.9.

By depth 3 we are done.

The dual principal graph has to be the same as the principal graph since $M \subset M_{1}$ satisfies the same hypotheses as $N \subset M$.

$$
\text { 6. The } 6+4 \sqrt{2} \text { EXAMPLE }
$$

6.1. Material from "Coxeter graphs and towers of algebras". We give a general construction for pairs of intermediate subfactors which seems to be of some interest. Recall two constructions of subfactors from [12]:

Let $\Gamma$ be a Coxeter-Dynkin diagram of type $A, D$ or $E$ with Coxeter number $k$, with $\Gamma=\Gamma_{0} \sqcup \Gamma_{1}$ a particular bipartite structure. Construct a pair $A_{0} \subset A_{1}$ of finite-dimensional $\mathrm{C}^{*}$-algebras the underlying graph of whose Bratteli diagram is $\Gamma$. Thus the minimal central projections in $A_{i}$ are indexed by $\Gamma_{i}$ for $i=0,1$. Using the Markov trace $\operatorname{tr}$ on $A_{1}$, iterate the basic construction to obtain the tower $A_{i+1}=\left\langle A_{i}, e_{i}\right\rangle, e_{i}$ being the orthogonal projection onto $A_{i-1}$. There is a unitary braid group representation inside the tower obtained by sending the usual generators $\sigma_{i}$ of the braid group (see [17]) to the elements $g_{i}=(t+1) e_{i}-1$ with $t=e^{2 \pi i / k}$.

First construction: commuting squares.

If we attempt to obtain a commuting square from the tower by conjugating $A_{1}$ inside $A_{2}$ by a linear combination of $e_{1}$ and 1 , we find that there are precisely two choices up to scalars: $g_{1}$ and $g_{1}^{-1}$. Then the following is a commuting square:

$$
\begin{array}{ccc}
B_{1}=g_{1} A_{1} g_{1}^{*} & \subset & A_{2} \\
B_{0}=A_{0} & \subset & A_{1}
\end{array}
$$

We may then define $B_{i}$ to be the $\mathrm{C}^{*}$-algebra generated by $B_{i-1}$ and $e_{i}$ to obtain $\mathrm{II}_{1}$ factors $B_{\infty} \subseteq A_{\infty}$ with index $4 \cos ^{2} \pi / k$. This construction is known to give all subfactors of index less than 4 of the hyperfinite $\mathrm{II}_{1}$ factor. The Dynkin diagram $\Gamma$ is the principal graph of the subfactor in the cases $A_{n}, D_{2 n}, E_{6}$ and $E_{8}$ but not otherwise. For $D_{2 n+1}$ the principal graph is $A_{4 n-1}$. See [10].

Second construction: GHJ subfactors. 
The $e_{i}$ 's in the $\mathrm{II}_{1}$ factor $A_{\infty}$ above generate a $\mathrm{II}_{1}$ factor $T L$ and by a lemma of Skau (see [12]) $T L^{\prime} \cap A_{\infty}=A_{0}$. Thus one may obtain irreducible subfactors $N \subseteq M$ by choosing a minimal projection $p$ in $A_{0}$, i.e. a vertex of $\Gamma$ in $\Gamma_{0}$, and setting $N=p T L$ and $M=p A_{\infty} p$. These subfactors are known as "GHJ" subfactors as they first appeared in [12. We will call the subfactor $T L \subseteq A_{\infty}$ the "full GHJ subfactor". The indices of the GHJ subfactors are all finite and were calculated in 12 (but note the error there: for $D_{n}$ using the two univalent vertices connected to the trivalent one, it should be divided by 2 ).

Remark 6.1 . The cut-down Temperley-Lieb projections $p e_{1}, p e_{2}, \ldots$ satisfy the same relations in the cut-down algebra $p A_{\infty} p$ that the projections $e_{1}, e_{2}, \ldots$ do in $A_{\infty}$. Therefore when discussing $p A_{\infty} p$ we will denote the cut-down Temperley-Lieb projections simply by $e_{i}$.

Using Skau's lemma, Okamoto in 29 calculated the principal graphs for the GHJ subfactors as follows: if $T L_{n}$ is the $\mathrm{C}^{*}$-algebra generated by $e_{1}, e_{2}, \ldots, e_{n-1}$, then the inclusions:

$$
\begin{array}{cccc}
p T L_{n+1} & \subset & p A_{n+1} p \\
\cup & & \cup \\
p T L_{n} & \subset & p A_{n} p
\end{array}
$$

are commuting squares for which the Bratteli diagram of the unital inclusion $p T L_{n} \subseteq p A_{n} p$ may be calculated explicitly inductively using one simple rule which follows from the basic construction.

Rule: If $q$ is a minimal projection in $p T L_{n}$ and $r$ is a minimal projection in $p A_{n} p$, then $e_{n+1} q$ and $e_{n+1} r$ are minimal projections in $p T L_{n+2}$ and $p A_{n+2} p$ respectively, and the number of edges connecting $q$ to $r$ is equal to the number connecting $e_{n+1} q$ to $e_{n+1} r$.

Thus one obtains two Bratteli diagrams depending on the parity of $n$. For sufficiently large $n$ the inclusion matrices for these Bratteli diagrams do not change and the principal graph for the GHJ subfactor is the underlying bipartite graph of the stable Bratteli diagram for the inclusion $p T L_{n} \subseteq p A_{n} p$, with distinguished vertex $*$ being the $*$ vertex in the Temperley-Lieb type $A$ graph. This specifies the parity of $n$ that is needed. Note that the dual principal graph is not in general the inclusion graph with the other parity!

Example 6.2. We take $\Gamma$ to be the Coxeter graph $D_{5}$ with the minimal projection $p$ being that corresponding to the trivalent vertex. The two vertical Bratteli diagrams are those for $p A_{\infty} p$ and $p T L$, and the inclusions $p T L_{n} \subset p A_{n} p$ are given by approximately horizontal heavy lines; the one which is the GHJ subfactor principal graph is made up of the heavy lines at the top of the figure. We have suppressed the heavy lines for $p T L_{5} \subset p A_{5} p$ to avoid confusion and because this inclusion graph is not the principal graph. The figure has been constructed from the bottom up 
using the basic construction and the above rule.

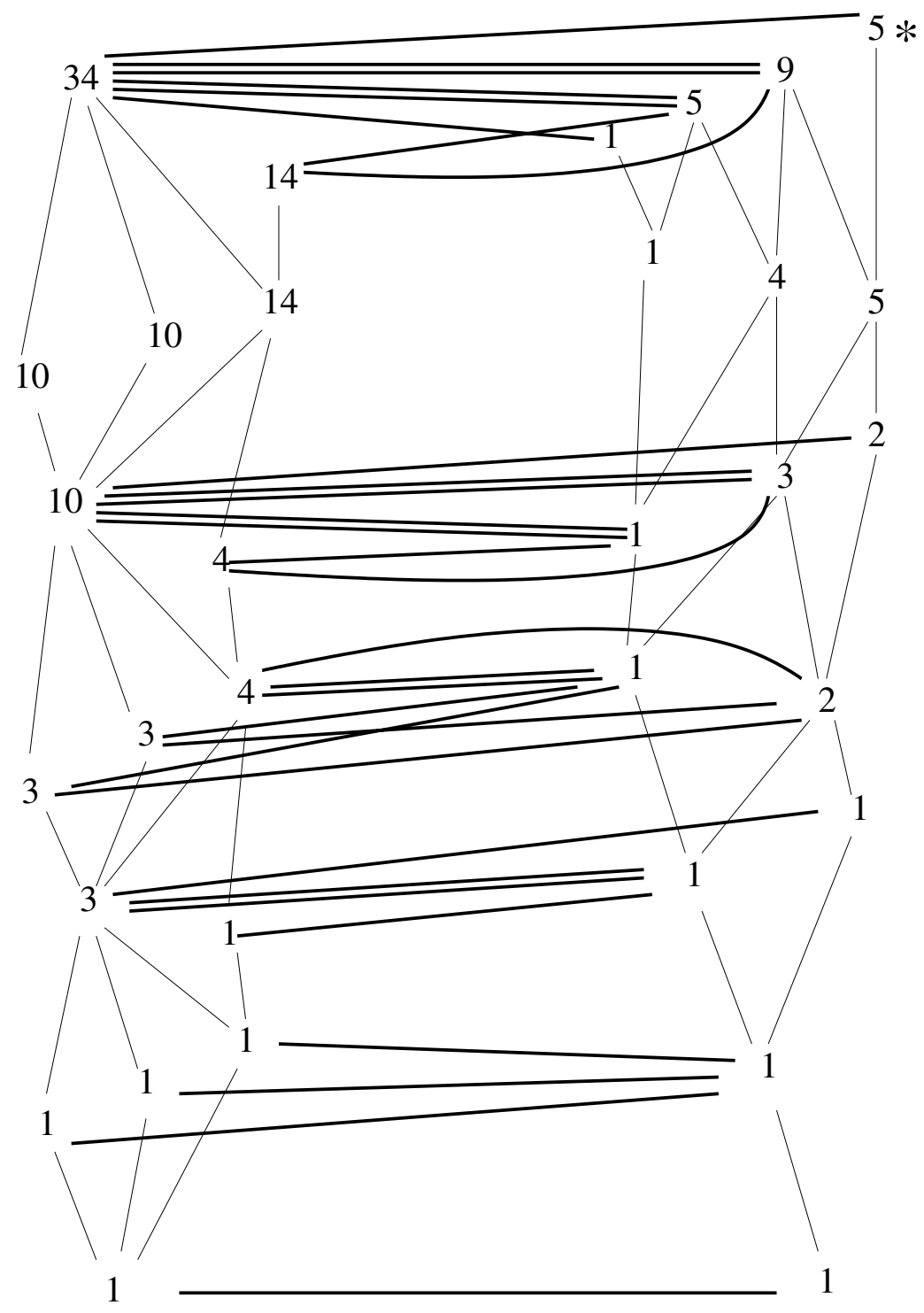

Making the principal graph more visible we obtain:

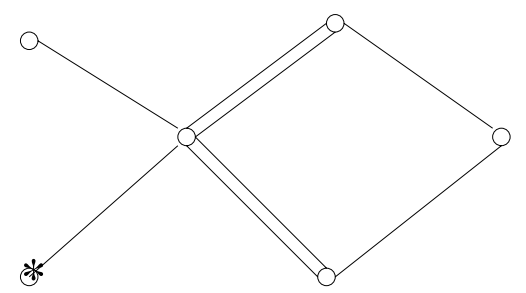

6.2. GHJ Subfactor pairs. Looking again at the commuting square construction from the original Coxeter-Dynkin diagram we see that we may in fact construct two subfactors of $A_{\infty}$ by conjugating initially by $g$ and $g^{-1}$ ! This construction 
works in great generality and gives a pair of subfactors whenever a subfactor is constructed using the endomorphism method of [12, 22]. In fact there is a way to obtain the quadrilateral with no extra structure by a simpler method, with simpler angle calculation and using only the real numbers. It seems to be a bit less general than the method using the braid group, so we present it second.

Definition 6.3. The full GHJ subfactor pair is the pair $\mathfrak{P}$ and $\mathfrak{Q}$ of subfactors of the (hyperfinite) $\mathrm{II}_{1}$ factor $A_{\infty}$ defined as the von Neumann algebras generated by the $P_{n}$ and $Q_{n}$ in the following towers:

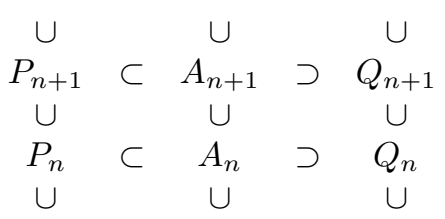

FIGURE 6.4.

where $A_{n}$ is as above, $P_{1}=Q_{1}=A_{0}, P_{2}=g_{1} A_{1} g_{1}^{*}, Q_{2}=g_{1}^{*} A_{1} g_{1}$ and $P_{n+1}=$ $\left\{P_{n}, e_{n}\right\}^{\prime \prime}, Q_{n+1}=\left\{Q_{n}, e_{n}\right\}^{\prime \prime}$.

Note that in Figure 6.4 all squares involving just $A$ 's and $P$ 's or just $A$ 's and $Q$ 's are commuting but squares involving $P$ 's and $Q$ 's may not be.

Definition 6.5. Let $T L 2$ be the subfactor of $A_{\infty}$ generated by all the $e_{i}$ with $i \geq 2$.

Proposition 6.6. $\left[A_{\infty}: \mathfrak{P} \cap \mathfrak{Q}\right]<\infty$.

Proof. By construction $e_{i} \in \mathfrak{P} \cap \mathfrak{Q}$ for all $i \geq 2$. Moreover $T L 2$ is of index $4 \cos ^{2} \pi / k$ in the full GHJ subfactor $T L$ which is in turn of finite index in $A$ by [12].

Note that $A_{0}$ is in $T L_{2}^{\prime} \cap A_{\infty}$ and $A_{0} \subseteq \mathfrak{P} \cap \mathfrak{Q}$. We suspect that $\mathfrak{P} \cap \mathfrak{Q}$ is the von Neumann algebra $T L 2 \otimes A_{0}$ generated by $T L 2$ and $A_{0}$. We hope to answer this question in a future systematic study of the GHJ subfactor pairs.

Our interest in this paper has been in pairs of subfactors $P, Q \subseteq M$ with $(P \cap Q)^{\prime} \cap M=\mathbb{C} i d$.

Definition 6.7. Let $p$ be a projection in $A_{0}$ that is minimal in $A_{1}$. Then the GHJ subfactor pair corresponding to $p$ is the pair of subfactors

$$
P=p \mathfrak{P} p, Q=p \mathfrak{Q} p \quad \subseteq \quad M=p A_{\infty} p .
$$

Proposition 6.8. If $P, Q \subseteq M$ is a GHJ subfactor pair, then $(P \cap Q)^{\prime} \cap M=\mathbb{C} i d$.

Proof. By Skau's lemma we know that the commutant of TL2 in $M$ is $A_{1}$.

A projection in $A_{0}$ that is minimal in $A_{1}$ is the same thing as a univalent vertex in $\Gamma_{0}$. Note that the subfactor $T L 2 \subseteq A_{\infty}$ is then the full GHJ subfactor for the other bipartite structure on $\Gamma$, and the subfactor $p T L 2 \subseteq p A_{\infty} p$ is the GHJ subfactor obtained by choosing the unique neighbour of the original univalent vertex. (This is because the inclusion $A_{1} \subseteq A_{2}$ can be used as the initial inclusion to construct the full GHJ subfactor for the other bipartite structure and $p$ is a minimal projection in $A_{1}$ since we started with a univalent vertex.) 
There are not too many choices for the univalent vertex, especially up to symmetry. We enumerate them below, the chosen univalent vertex being indicated with a $*$ :
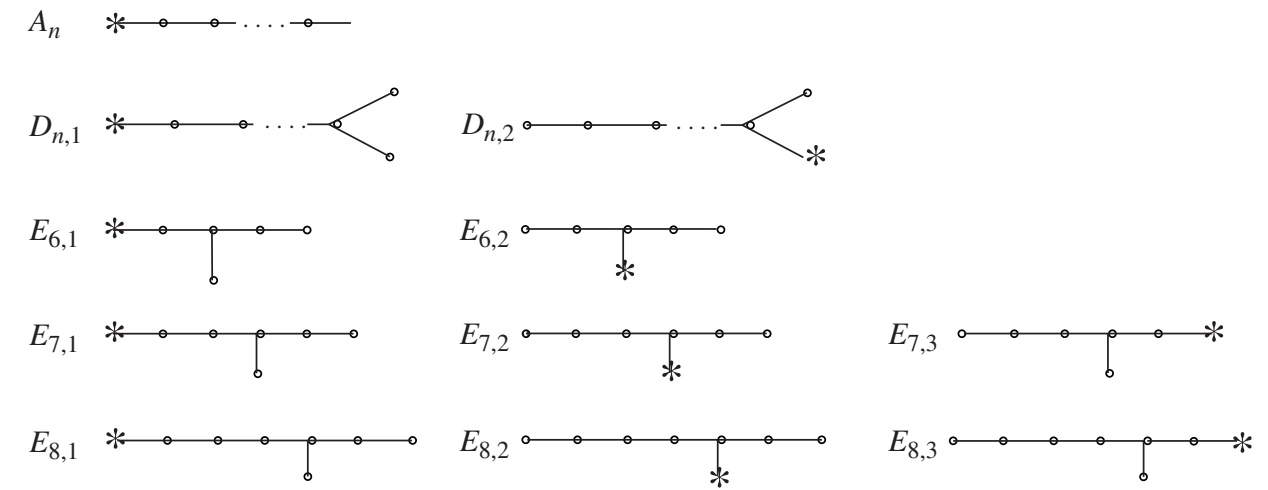

Proposition 6.9. The subfactor $p T L 2 \subseteq M$ in the case $D_{5,2}$ has index $(2+\sqrt{2})^{2}$ and principal graph

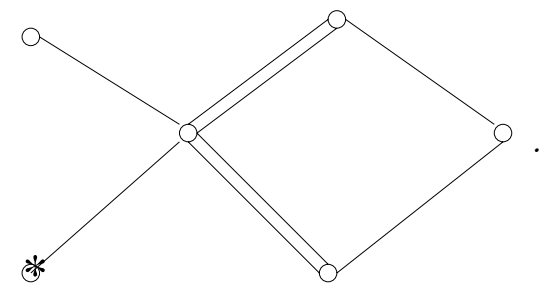

Proof. This is just the calculation done in Example 6.2

At this stage it looks very likely that the $D_{5,2}$ pair realises the case in Theorem 5.8 of a no-extra-structure quadrilateral. In order to be sure of this we need to know that $P$ and $Q$ in this case do not commute. To do this we shall compute the angle between them. At this stage we do not even know if $P$ and $Q$ are distinct.

6.3. Angle computation. Our strategy for calculating the angle between $P$ and $Q$ will work whenever the subfactors $T L 2 \subseteq P$ and $T L 2 \subseteq Q$ are 2-transitive. So in this subsection we only assume that of the Coxeter graph with chosen univalent vertex.

Definition 6.10. Let $\Gamma$ be a pointed Coxeter graph of type $D_{n}$ for $n>4$ or $E$ on the list above. Then $d=d(\Gamma)$ will denote the distance from ${ }^{*}$ to the trivalent vertex.

Thus $d\left(E_{6,1}\right)=2$ and $d\left(D_{5,2}\right)=1$.

Theorem 6.11. Suppose $\Gamma$ to be a pointed Coxeter graph of type $D_{n}$ for $n>4$ or $E$, with Coxeter number $\ell$, and that the GHJ subfactor with the starred vertex is 2-transitive. Then the angle between the two intermediate subfactors is

$$
\left\{0, \pi / 2, \cos ^{-1}\left(\left|\frac{\cos (2 d+3) \pi / \ell}{\cos \pi / \ell}\right|\right)\right\} .
$$


Proof. The idea is as follows: by 2-transitivity, $E_{P} E_{Q} E_{P}$ is a multiple of the identity on the orthogonal complement of $T L 2$ in $P$, so it suffices to find an element $x$ of this orthogonal complement and calculate $\left\|E_{Q}(x)\right\|_{2}$. We will find our element $x$ in $p P_{d+2} p$, which is the smallest $p P_{k} p$ that is strictly larger than $p T L 2_{k}$. It will be convenient to pull back the calculations to $p A_{n} p$. So in the next lemma we give the unitaries which conjugate $A_{n}$ to $P_{n+1}$ and $Q_{n+1}$. These unitaries may be deduced from [12] but we give a proof here for the convenience of the reader.

Lemma 6.12. Let $v_{n}=g_{1} g_{2} \ldots g_{n}$ and $w=g_{1}^{-1} g_{2}^{-1} \ldots g_{n}^{-1}$. Then

(a) $P_{n+1}=v_{n} A_{n} v_{n}^{*}$ and $Q_{n+1}=w_{n} A_{n} w_{n}^{*}$;

(b) $T L 2_{n}=v_{n} T L_{n} v_{n}^{*}=w_{n} T L_{n} w_{n}^{*}$.

Proof. Braid group relations give $v_{n} g_{i} v_{n}^{*}=g_{i+1}$ and $w_{n} g_{i}^{-1} w_{n}^{*}=g_{i+1}^{-1}$; hence $v_{n} e_{i} v_{n}^{*}=e_{i+1}$ and $w_{n} e_{i} w_{n}^{*}=e_{i+1}$ for $1 \leq i \leq n-1$. This proves the assertion (b) about the Temperley-Lieb algebras. Since $\left[e_{i}, A_{1}\right]=0$ for $i \geq 2$ we get $v_{n} A_{1} v_{n}^{*}=g_{1} A_{1} g_{1}^{*}=P_{1}$ and $w_{n} A_{1} w_{n}^{*}=Q_{1}$. By the definition of $P_{n}$ and $Q_{n}$ we are done.

As in Example 6.2 the Bratteli diagram for $p A_{\infty} p$ is given by taking the full Bratteli diagram for $A_{\infty}$ and considering only edges emanating from the starred vertex. Thus by the definition of $d(\Gamma)$ there is an element $y$ of $p A_{d+1} p$ which is orthogonal to $T L_{d}$ and is unique up to a scalar multiple. We may assume $\|y\|_{2}=1$ and $y=y^{*}$. Define $x \in P_{d+2}$ by $x=v_{d+1} y v_{d+1}^{*}$. By Lemma 6.12 we know that $x$ is orthogonal to $e_{2}, e_{3}, \ldots, e_{d+1}$. Moreover since $\operatorname{tr}(x)=0$ (since $\left.x \perp 1\right), E_{P_{d+1}}(x)=0$ so $e_{d+2} x e_{d+2}=0$ and taking the trace, $x \perp e_{d+2}$. By the usual properties of the Markov trace in a tower, $x \perp e_{n}$ for $n>d+2$. Thus $x \perp T L 2$.

Since the inclusions of $p Q_{n} p$ in $p A_{n} p$ are commuting squares we may calculate $E_{Q}(x)$ by $E_{p Q_{d+2} p}(x)$ (inside $p A_{d+2} p$ ). But this element of $p Q_{d+2} p$ is orthogonal to $T L 2$, so is a multiple of $w_{d+1} y w_{d+1}^{*}$. So the cosine of the angle between $P$ and $Q$ is the absolute value of the inner product

$$
\operatorname{tr}\left(x w_{d+1} y w_{d+1}^{*}\right)=\operatorname{tr}\left(v_{d+1} y v_{d+1}^{*} w_{d+1} y w_{d+1}^{*}\right) .
$$

The algebras $p A_{n} p$ are all included in the planar algebra for the bipartite graph $\Gamma$ as defined in 20], so we may use the diagrams therefrom. In particular the inner product we need to calculate is given by the partition function in Figure 6.13 (up to a power of $\delta=2 \cos \pi / \ell$ ).

The crossings in Figure 6.13 are the braid elements $g_{i}$ with some convention as to which is positive and which is negative, read from bottom to top. We have illustrated with $d=2$ for concreteness. They may be evaluated using the Kauffman picture:

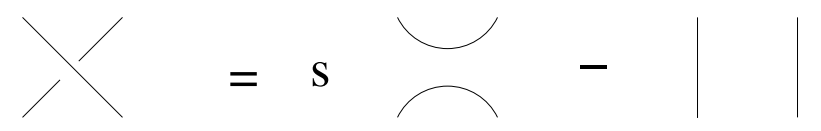

where $s=e^{\pi i / \ell}$. 


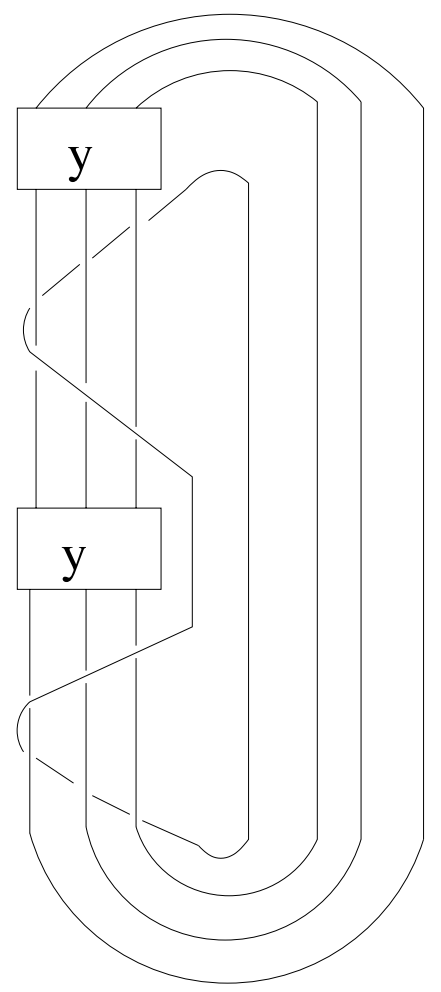

FIGURE 6.13.

The orthogonality of $y$ to $T L$ is equivalent to the fact that, if any tangle contains a $y$ box with two neighbouring boundary points connected by a planar curve (in which case we say the box is "capped off"), the answer is zero. Thus one may evaluate Figure 6.13 as follows.

Using the Kauffman relation in Figure 6.13 inside the dotted circle one obtains Figure 6.14

Consider the first diagram on the right-hand side of the equation in Figure 6.14, Following the curve in the direction indicated by the arrow, observe that one choice of the two possibilities in applying the Kauffman relation at each crossing always results in one of the $y$ boxes being capped off. The first $d$ such crossings thus contribute a factor of $s$ each. Then one meets the situation $\lambda$ which is easily seen to be the same as $s^{2}$ times $\bigcirc$ One then meets $d$ more crossings, each of which contributes $s$. After this (the crossings below the bottom $y$ box in Figure 6.13) the only contributing terms in the Kauffman relation just give the sign -1 . Since there are an even number of them we deduce that the diagram of the first term on the right-hand side of Figure 6.14 is $s^{2 d+2}$ times a tangle which is $\operatorname{tr}\left(y^{2}\right)$ up to a power of $\delta$. A similar analysis of the diagram of the second term gives $-s^{-(2 d+3)}$ times 

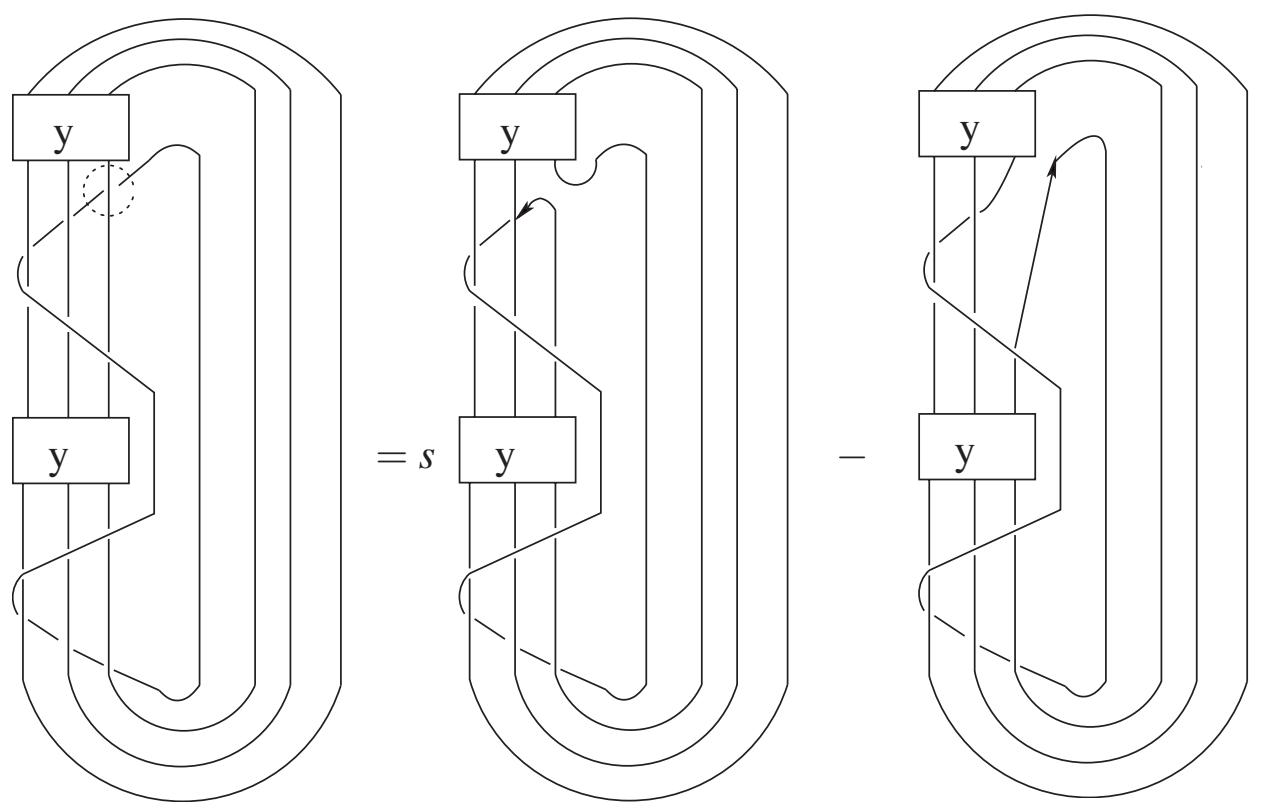

FiguRe 6.14 .

$\operatorname{tr}\left(y^{2}\right)$. A little thought concerning the powers of $\delta$ gives the final result that

$$
\operatorname{tr}\left(v_{d+1} y v_{d+1}^{*} w_{d+1} y w_{d+1}^{*}\right)=\frac{s^{2 d+3}+s^{-2 d-3}}{s+s^{-1}} .
$$

This ends the proof of Theorem 6.11

Corollary 6.15. For the GHJ subfactor pair given by $D_{5,2}$, there is no extra structure, and the angle between $P$ and $Q$ is $\cos ^{-1}(\sqrt{2}-1)$, and $P \cap Q=T L_{2}$.

Proof. We have $[M: P]=4 \cos ^{2} \pi / 8$ from the $D_{5}$ commuting square. Also $p T L 2 \subseteq$ $P$ has the same index from a GHJ calculation, or from the one already done for $D_{5}$. So there cannot be subfactors between $p T L 2$ and $P$ or $Q$, and $p T L 2 \subseteq P$ is 2 -transitive. So we can apply the previous theorem to get the angle. The only possible principal graph with index $4 \cos ^{2} \pi / 8$ is $A_{7}$, so there is no extra structure.

6.4. A simpler quadrilateral with no extra structure. Note that the definition of the GHJ pair will require the use of certain roots of unity. But at least in the $D_{n, 2}$ case it is possible to find another pair $\tilde{P}$ and $\tilde{Q}$ between $p T L 2$ and $M$, which is defined over $\mathbb{R}$ ! We will see that both $\tilde{P}$ and $\tilde{Q}$ form commuting cocommuting squares with both $P$ and $Q$. One of these two intermediate subfactors is quite canonical and exists whenever $P \cap Q=T L_{2}$.

Definition 6.16. Let $\Gamma$ etc. be as above. Let $\tilde{P}$ be the GHJ subfactor for $p$, i.e. the subfactor generated by $p T L 2$ and $p e_{1}$. 
Proposition 6.17. The quadrilaterals $N \subset \tilde{P}, P \subset M$ and $N \subset \tilde{P}, Q \subset M$ are commuting squares.

Proof. Reducing by $p$ is irrelevant, so we can do the computation in the full GHJ factor. As in the proof of Theorem 6.11 it suffices to find a nonzero element of $\tilde{P}$ orthogonal to $T L 2$ and show that its projection onto $P$ is zero. Let $x=e_{1}-$ $\tau i d$, where $\tau=\left(4 \cos ^{2} \pi / \ell\right)^{-1}$. Then since the $P_{n}$ 's form commuting squares with the $A_{n}$ 's and $e_{1} \in A_{2}$ we need only project onto $P_{2}=A d g_{1}\left(A_{1}\right)$. But $E_{P_{2}}=$ $A d g_{1} E_{A_{1}} A d g_{1}^{-1}$ and $A d g_{1}(x)=x$. But $E_{A_{1}}(x)=0$ is just the Markov property for the trace on $A_{2}$. The same argument applies to $\tilde{Q}$.

Lemma 6.18. Let $\Gamma$ be $D_{n, 2}$ for $n \geq 5$. Then there is a projection $f$ in $p A_{2}$ with the following properties:
(a) $\operatorname{tr}(f)=\tau$;
(b) $\mathrm{fpe}_{1}=0$;
(c) $p e_{2} f p e_{2}=\tau e_{2}$ and $f p e_{2} f=\tau f$.

Proof. From the Bratteli diagram for $p A_{2}$, it has three minimal projections, which are central. One is clearly $p e_{1}$ and one of the other two has the same trace by symmetry. Let $f$ be that other one. Then (a) and (b) are obvious. The first part of (c) follows from $\operatorname{dim}\left(p A_{1}\right)=1$ and the second part follows since, from the Bratteli diagram, $f$ is a minimal projection in $p A_{3}$.

Definition 6.19. Let $\Gamma$ be $D_{n, 2}$ for $n \geq 5$. Let $\tilde{Q}$ be the von Neumann algebra generated by $p T L_{2}$ and the $f$ of Lemma 6.18 .

Theorem 6.20. Let $\Gamma$ be $D_{n, 2}$ for $n \geq 5$. Then $\tilde{Q}$ is a $\mathrm{II}_{1}$ factor with $\left[\tilde{Q}: p T L_{2}\right]=$ $4 \cos ^{2} \pi / \ell$, and the angle between $\tilde{P}$ and $\tilde{Q}$ is $\cos ^{-1}\left(\frac{\tau}{1-\tau}\right)$.

Proof. Lemma 6.18 and the properties of the basic construction show that $f$ has exactly the same commutation relations and trace properties with $p e_{i}$ for $i \geq 2$ as does $p e_{1}$. Thus by $16, \tilde{Q}$ is a $\mathrm{II}_{1}$ factor with the given index. Moreover the subfactor $p T L_{2} \subset \tilde{Q}$ is 2-transitive, so we can speak of the angle between $\tilde{P}$ and $\tilde{Q}$.

The angle calculation is not hard. As in Theorem $\overline{6.11}$ it suffices to compute the length of the projection onto $\tilde{P}_{1}$ of a unit vector in $\tilde{Q}$ orthogonal to $p T L_{2}$. By Lemma 6.18, the element $x=f-\tau i d$ is orthogonal to the two-dimensional algebra $p T L_{2}$ and $\operatorname{tr}\left(x^{*} x\right)=\tau(1-\tau)$. Since the $p T L_{n}$ form commuting squares with the $p A_{n}, E_{\tilde{P}}(x)$ is just the projection $E(x)$ of $x$ onto $p T L_{2}$. By the bimodule property of $E, E(x) p e_{1}=-\tau p e_{1}$, so $E(x)=\tau p e_{1}+\lambda\left(p-p e_{1}\right)$. Using $\operatorname{tr}(x)=0$ we find $\lambda=-\tau^{2} /(1-\tau)$. So

$$
\|E(x)\|^{2}=\tau^{3}+\left(\frac{\tau^{2}}{1-\tau}\right)^{2}(1-\tau)=\frac{\tau^{3}}{1-\tau},
$$

and finally,

$$
\frac{\|E(x)\|^{2}}{\|x\|_{2}^{2}}=\frac{\tau^{2}}{(1-\tau)^{2}}
$$


Observe that for $\tau^{-1}=4 \cos ^{2} \pi / \ell, \tau /(1-\tau)=\sqrt{2}-1$, so the angle between $\tilde{P}$ and $\tilde{Q}$ is indeed the same as that between $P$ and $Q$, and the quadrilateral formed by $\tilde{P}$ and $\tilde{Q}$ has no extra structure for the same reasons as the one formed by $P$ and $Q$. As a last detail observe that the quadrilaterals $N \subset \tilde{Q}, P \subset M$ and $N \subset \tilde{Q}, Q \subset M$ are commuting squares. We leave the argument to the reader.

\section{UNIQUENESS}

Outer actions of finite groups are extremely well understood, so we need say nothing more in the case $[M: N]=6$. Uniqueness up to conjugacy in the hyperfinite case follows from 15 .

So from now on we assume that $[M: N]=6+4 \sqrt{2}$ and that there are two intermediate subfactors $P$ and $Q$ which neither commute nor cocommute. We will eventually show that all the constants in a planar algebra presentation of the standard invariant of $N \subseteq M$ are determined by this data.

From the structure of the principal graph we see that there is exactly one projection in $N^{\prime} \cap M_{1}$ different from $e_{1}$ but with the same trace as $e_{1}$. By [30] this means that there is a self-adjoint unitary in the normaliser of $M$ in $M_{1}$ (and in the normaliser of $M_{1}$ in $M_{2}$ ). We record some useful diagrammatic facts about normalisers below. It is convenient to work with the normaliser of $M_{1}$ in $M_{2}$, but any subfactor is dual, so the result can be modified for the normaliser of $M$.

7.1. Diagrammatic relations for the normaliser. If $N \subseteq M$ is an irreducible finite index subfactor, then we will consider an element $u$ in the normaliser of $M_{1}$ inside $M^{\prime} \cap M_{2}$, that is to say, a unitary in $M^{\prime} \cap M_{2}$ with $u M_{1} u^{*}=M_{1}$. First observe that such a unitary defines an automorphism $\alpha$ of $M_{1}$ by $\alpha(x)=u x u^{*}$.

Proposition 7.1. $\alpha(x)=x$ for all $x \in M$.

Proof. This follows immediately from $u \in M^{\prime}$.

The automorphism $\alpha$ in turn defines a unitary on $L^{2}\left(M_{1}\right)$ which is in $M^{\prime} \cap M_{2}$ and by irreducibility differs from $u$ by a scalar. Thus we may alter $u$ so that $u=\alpha$ as maps on $L^{2}\left(M_{1}\right)$. The element $u$ is in $N^{\prime} \cap M_{2}$, so in the planar algebra picture it may be represented by a diagram:

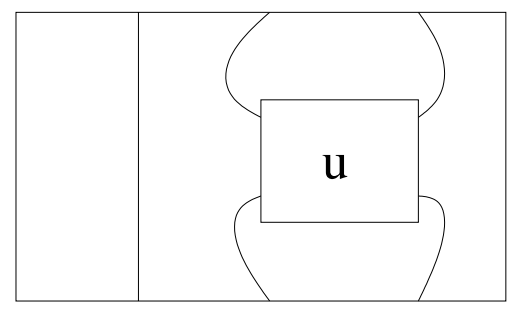

and the relation $u x u^{*}=\alpha(x)$ for $x \in N^{\prime} \cap M_{1}$ is the equality in Figure 7.2 . 


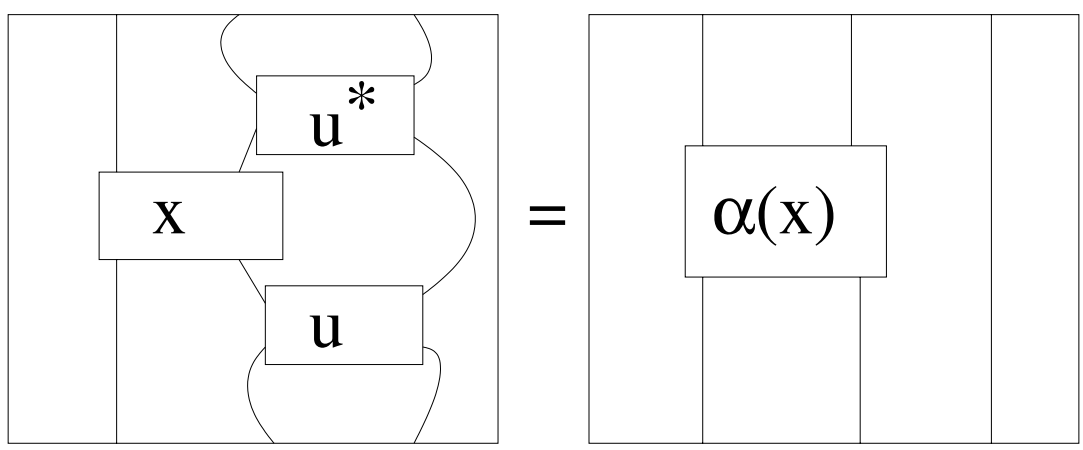

FiguRE 7.2.

We will make considerable use of the following result:

Lemma 7.3. If $u=u^{*}$ is in the normaliser as above, then

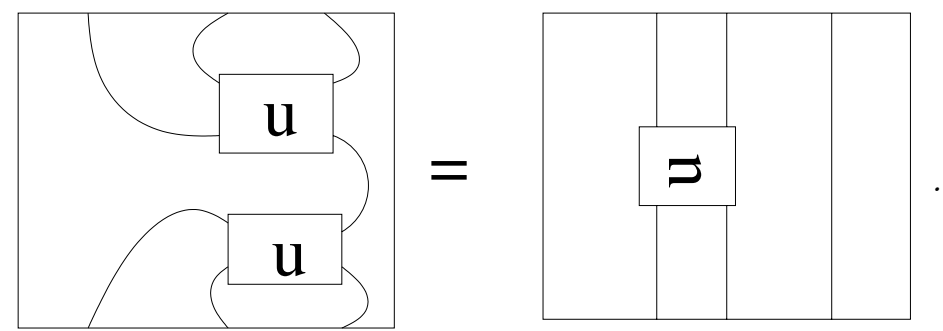

Proof. We first establish the result for any $u$ in the normaliser with $u=\alpha$ as above, and $x \in N^{\prime} \cap M_{1}$ (see Figure 7.4).

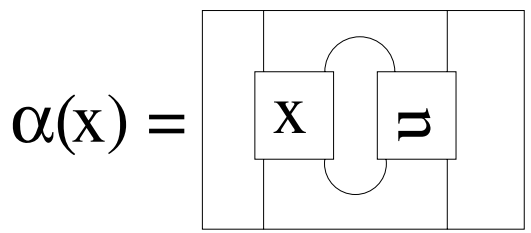

FIGURE 7.4. 
For this observe that if $a=x e_{2} y$ for $x, y \in M_{1}$ and $b \in M_{1} \subseteq L^{2}\left(M_{1}\right)$, $E_{M_{1}}\left(a b e_{2}\right)=\delta^{-2} x E_{M}(y b)=a(b)$. Since linear combinations of elements of the form $x e_{2} y$ span $M_{2}$ we have

$$
E_{M_{1}}\left(a b e_{2}\right)=\delta^{-2} a(b)
$$

for all $a \in M_{2}$ and $b \in M_{1}$. Drawing this relation diagramatically for $a=u$ and $b=x$ in $N^{\prime} \cap M_{1}$ we obtain the diagram for $\alpha(x)$. Finally apply Figure 7.2 with $x=e_{1}$ and the above diagram to obtain the lemma.

Corollary 7.5. With notation as above, $u$ is a coprojection.

Proof. Use the property that $\alpha$ is a $*$-automorphism in the previous lemma.

7.2. The structure of $N^{\prime} \cap M_{1}$. We need to adopt some conventions for the position of certain operators in $N^{\prime} \cap M_{1}$. Since the angle between $P$ and $Q$ consists of one value (different from $0, \pi / 2$ ), we know that $e_{P}$ and $e_{Q}$ generate a $2 \times 2$ matrix algebra modulo $e_{N}$. We also know from the dual principal graph that there is an intermediate subfactor $S$ with $[S: N]=2$. If $e_{S}$ is the projection onto $S$, then the trace of $e_{S}$ is $\frac{2}{6+4 \sqrt{2}}$ and it is $e_{N}$ plus a minimal projection in $N^{\prime} \cap M_{1}$. This means that $e_{S}$ must be orthogonal to both of the $2 \times 2$ matrix algebras in $N^{\prime} \cap M_{1}$ since the traces of minimal projections therein do not match.

Definition 7.6. We write $N^{\prime} \cap M_{1}=e_{N} \mathbb{C} \oplus A \oplus B \oplus\left(e_{S}-e_{N}\right) \mathbb{C}$, where $A$ and $B$ are $2 \times 2$ matrix algebras with $e_{P} A \neq 0$.

This definition specifies $A$ uniquely since $\operatorname{tr}\left(e_{P}\right)=(2+\sqrt{2})^{-1}, \operatorname{tr}\left(E_{N}\right)=$ $(2+\sqrt{2})^{-2}$ and the trace of a minimal projection in $A$ is $\frac{1+\sqrt{2}}{(2+\sqrt{2})^{2}}$. Thus $e_{P} B=0$.

7.3. Relations between elements in $N^{\prime} \cap M_{1}$. From Theorem 5.8 we know that the principal and dual principal graphs are the same and that there is a single projection of trace equal to that of $e_{N}$ in all the (second) relative commutants. This means by [30] that for each inclusion $M_{i} \subset M_{i+1}$ there is an intermediate inclusion $R_{i}$ with $\left[R_{i}: M_{i}\right]=2$. By duality there are thus $S_{i}$ with $M_{i} \subset S_{i} \subset M_{i+1}$ so that $S_{i} \subset M_{i+1} \subset R_{i+1}$ is a fixed point/crossed product pair for an outer action of $\mathbb{Z} / 2 \mathbb{Z}$. In particular there are unitaries $u_{i}$ satisfying the conditions of the previous section at every step in the tower. So let $\alpha$ be the period two automorphism of $M$ (which is the identity on $N$ ) defining an element $u$ of $N^{\prime} \cap M_{1}$. Then $\frac{u+1}{2}$ is the projection onto an intermediate subfactor of index 2 for $N \subset M$ which we shall call $R$. Thus

$$
[M: R]=2 \text { or } \operatorname{tr}\left(e_{R}\right)=\frac{1}{2}, \quad \text { and } u=2 e_{R}-1 .
$$

Lemma 7.7. The subfactors $P$ and $R$ cocommute but do not commute, $e_{P} e_{R} e_{P}=$ $e_{N}+\left(1-\frac{1}{\sqrt{2}}\right)\left(e_{P}-e_{N}\right)$ and $e_{R} B \neq 0$.

Proof. Since $L^{2}\left(M_{1}\right) \cong U_{0} \oplus 2 U_{1} \oplus 2 U_{2} \oplus U_{3}$ as $M-M$ bimodules, where $L^{2}(\bar{P}) \cong$ $U_{0} \oplus U_{1}$ and $L^{2}(\bar{R}) \cong U_{0} \oplus U_{3}$, the dual subfactors $\bar{P}$ and $\bar{R}$ commute. However, $[\bar{P}: M][\bar{R}: M]<\left[M_{1}: M\right]$, so by Lemma 3.18, $\bar{P}$ and $\bar{R}$ do not cocommute. Thus $P$ and $R$ cocommute but do not commute. Then $L^{2}(R)$ must be of the form $V_{0} \oplus V_{1} \oplus V_{2}$, so $e_{R} B \neq 0$. Since $N \subset P$ is 2 -supertransitive, by Lemma 4.14 we have

$$
e_{P} e_{R} e_{P}=e_{N}+\frac{\operatorname{tr}\left(e_{\bar{P} \bar{R}}\right)^{-1}-1}{[P: N]-1}\left(e_{P}-e_{N}\right)
$$


Since the dual quadrilateral commutes, by Corollary 3.19 we have

$$
\operatorname{tr}\left(e_{\bar{P} \bar{R}}\right)=\frac{[\bar{P}: M][\bar{R}: M]}{[M: N]}=\frac{2}{2+\sqrt{2}} .
$$

Combining these equations gives the result.

We want to investigate the algebraic and diagrammatic relations between $e_{P}, e_{Q}$ and $u$. First we give a simple but crucial computation:

Lemma 7.8. $\operatorname{tr}\left(u e_{P}\right)=\operatorname{tr}\left(u e_{q}\right)=0$.

Proof. Since $P$ and $R$ cocommute, by Proposition 3.14, $\operatorname{tr}\left(e_{P} e_{R}\right)=\operatorname{tr}\left(e_{P}\right) \operatorname{tr}\left(e_{R}\right)=$ $1 / 2 \operatorname{tr}\left(e_{P}\right)$, and $u=2 e_{R}-1$.

We will use on several occasions the following result, which is no doubt extremely well known. We include a proof for the convenience of the reader.

Lemma 7.9. Let $P, Q, R, S$ be distinct projections onto four one-dimensional subspaces of $\mathbb{C}^{2}$ all making the same angle with respect to one another. Then that angle is $\cos ^{-1} \frac{1}{\sqrt{3}}$.

Proof. If we choose a basis so that

$$
P=\left(\begin{array}{ll}
1 & 0 \\
0 & 0
\end{array}\right),
$$

then any other projection at $\cos ^{-1}(\sqrt{a})$ to $P$ is of the form

$$
P=\left(\begin{array}{cc}
a & \omega \sqrt{a(1-a)} \\
\omega^{-1} \sqrt{a(1-a)} & 1-a
\end{array}\right),
$$

where $|\omega|=1$. Equating $a$ to the traces of $Q R, R S$ and $Q S$ we see that $\omega$ must be a proper cube root of unity and that $3 a^{2}-4 a+1=0$.

Corollary 7.10. $u e_{P} u=e_{Q}$ and $u e_{P Q} u=e_{Q P}$.

Proof. These are equivalent to $\alpha(P)=Q$. By Lemma 7.7, $u e_{P} u \neq P$. If $\alpha(P)$ were not equal to $Q$, then $P, Q, \alpha(P)$ and $\alpha(Q)$ are four distinct intermediate subfactors. But $u e_{P} u=e_{\alpha(P)}$ and $u e_{Q} u=e_{\alpha(Q)}$, so the $N-N$ bimodules defined by these four intermediate subfactors are all isomorphic to $L^{2}(P)$ and none of them commutes with any other. By Lemma 7.7, which guarantees that $\alpha(P)$ and $P$ do not commute, the angles between all four subfactors are the same and, by Corollary 5.7, equal to $\cos ^{-1}(\sqrt{2}-1)$. By Lemma 7.9, this is impossible.

Corollary 7.11. $u e_{P}=e_{N}+\frac{1}{1-\sqrt{2}}\left(e_{Q} e_{P}-e_{N}\right) ; u e_{Q}=e_{N}+\frac{1}{1-\sqrt{2}}\left(e_{P} e_{Q}-e_{N}\right)$.

Proof. $u\left(e_{P}-e_{N}\right)$ and $e_{Q}\left(e_{P}-e_{N}\right)$ are in $A$, and both are multiples of a partial isometry with intial domain $e_{P}-e_{N}$ and final domain $e_{Q}-e_{N}$. They are thus proportional. Taking the trace we get the result using Lemma 7.8 and Corollary 5.7.

This yields a different derivation of the angle between $P$ and $Q$. We see that modulo the ideal, $\mathbb{C} e_{N}$, we have $u e_{P}=\frac{1}{1-\sqrt{2}} e_{Q} e_{P}$ so that mod this ideal $e_{P}=$ $e_{P} u_{u} e_{P}=\left(\frac{1}{1-\sqrt{2}}\right)^{2} e_{P} e_{Q} e_{P}$, which determines the constant in the angle formula $e_{P} e_{Q} e_{P}-e_{N}=\operatorname{constant}\left(e_{Q}-e_{N}\right)$. 
Corollary 7.12. The identity $1_{A}$ of the $2 \times 2$ matrix algebra $A \subseteq N^{\prime} \cap M_{1}$ is $\frac{\sqrt{2}+1}{2}\left(e_{P}+e_{Q}\right)+1 / 2\left(u e_{P}+u e_{Q}\right)-(2+\sqrt{2}) e_{N}$.

Proof. From Corollary 5.7

$$
\left(e_{P}-e_{N}\right)\left(e_{Q}-e_{N}\right)\left(e_{P}-e_{N}\right)=(\sqrt{2}-1)^{2}\left(e_{P}-e_{N}\right) .
$$

So $1_{A}=\frac{\sqrt{2}+1}{2}\left(e_{P}-e_{Q}\right)^{2}$. Corollary 7.11 gives $e_{P} e_{Q}=\sqrt{2} e_{N}+(1-\sqrt{2}) u e_{Q}$, hence the result.

Lemma 7.13. $\operatorname{tr}\left(u e_{P Q}\right)=0$.

Proof. Since $u=2 e_{R}-1, \operatorname{tr}\left(u e_{P Q}\right)=2 \operatorname{tr}\left(e_{R} e_{P Q}\right)-1 / \sqrt{2}$ by Corollary 5.7. But $\operatorname{tr}\left(e_{R} e_{P Q}\right)$ is given by $\frac{1}{\delta^{3} \operatorname{tr}\left(e_{P} e_{Q}\right)}$ times the following diagram:

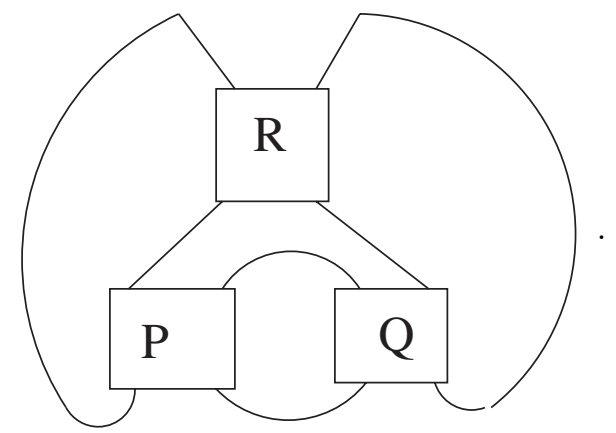

This is essentially the cotrace of $e_{R} \circ e_{P} \circ e_{Q}$, and we know that $e_{R} \circ e_{P}$ is $(2+$ $\sqrt{2}) \operatorname{tr}\left(e_{R}\right) \operatorname{tr}\left(e_{P}\right) i d$ by Theorem 3.10 since $P$ and $R$ cocommute. Using this in the figure we obtain

$$
\operatorname{tr}\left(e_{R} e_{P Q}\right)=\frac{1}{\delta^{3} \operatorname{tr}\left(e_{P} e_{Q}\right)}(2+\sqrt{2}) \operatorname{tr}\left(e_{R}\right) \operatorname{tr}\left(e_{P}\right) \delta^{2} \operatorname{tr}\left(e_{Q}\right)=\frac{1}{2 \sqrt{2}} .
$$

Lemma 7.14. $\operatorname{tr}\left(e_{P Q} e_{Q P}\right)=\frac{5 \sqrt{2}-6}{2}$.

Proof. As in Lemma 3.17 we recognise $\operatorname{tr}\left(e_{P Q} e_{Q P}\right)$ as being $\frac{1}{2[M: N]}$ times the cotrace of $e_{P} \circ e_{Q} \circ e_{P} \circ e_{Q}$. But since $[M: P]=[P: N], e_{P}$ and $e_{Q}$ are coprojections and the angles between them as coprojections are the same as the angles between them as projections. So $\operatorname{tr}\left(e_{P Q} e_{Q P}\right)=\frac{1}{2} \operatorname{tr}\left(\left(e_{P} e_{Q} e_{P}\right)^{2}\right)$. However from Corollary 5.7 $e_{P} e_{Q} e_{P}=e_{N}+\frac{\sqrt{2}-1}{\sqrt{2}+1}\left(e_{P}-e_{N}\right)$. Squaring and taking the trace gives the answer.

Corollary 7.15. $u e_{P Q}=e_{N}+u 1_{A}-(\sqrt{2}+1)\left(e_{Q P} e_{P Q}-\left(e_{N}+1_{A}\right)\right)$.

Proof. As in Corollary 7.11, $u\left(e_{P Q}-e_{N}-1_{A}\right)$ and $e_{Q P} e_{P Q}-e_{N}-1_{A}$ are both in $B$ (certainly $e_{P Q}>e_{Q}$ and the trace of $e_{P Q}$ is the trace of $e_{N}$ plus 3 times the trace of a minimal projection in $A$ so that $e_{P Q} e_{S}=0$ ) and are multiples of the same partial isometry. Taking the trace using the last two lemmas we get $u\left(e_{P Q}-e_{N}-1_{A}\right)=\frac{3+2 \sqrt{2}}{\sqrt{2}-1}\left(e_{Q P} e_{P Q}-e_{N}-1_{A}\right)$ and the result follows.

Corollary 7.16. $e_{P Q} e_{Q P} e_{P Q}-1_{A}-e_{N}=(\sqrt{2}-1)^{2}\left(e_{P Q}-1_{A}-e_{N}\right)$. 
Proof. Modulo the ideal spanned by $e_{N}$ and $A, u e_{P Q}=-(\sqrt{2}+1) e_{Q P} e_{P Q}$. So mod this ideal, $e_{P Q} u u e_{P Q}=(\sqrt{2}+1)^{2} e_{P Q} e_{Q P} e_{P Q}$. The left-hand and right-hand sides are proportional, and this determines the constant.

Taking the trace of this equality provides a useful check on our calculations. It is curious that $e_{P Q}$ and $e_{Q P}$ make the same angles as $e_{P}$ and $e_{Q}$.

\subsection{A basis and its structure constants.}

Definition 7.17. Let $\mathfrak{C}=\left\{e_{N}, 1\right\} \cup \mathfrak{A} \cup \mathfrak{B}$ where $\mathfrak{A}=\left\{e_{P}, e_{Q}, u e_{P}, u e_{Q}\right\}$ and $\mathfrak{B}=\left\{e_{P Q}, e_{Q P}, u e_{P Q}, u e_{Q P}\right\}$.

Theorem 7.18. $\mathfrak{C}$ is a basis for $N^{\prime} \cap M_{1}$, and all multiplication and comultiplication structure constants for this basis are determined.

Proof. That $\mathfrak{C}$ is a basis follows easily from the previous results: $\left\{e_{N}\right\} \cup \mathfrak{A}$ is a basis for $\mathbb{C} e_{N} \oplus A$ by Corollary 7.11 and $(2 \times 2)$-matrix calculations. Similarly $\mathfrak{B}$ forms a basis for $B$ modulo $\mathbb{C} e_{N} \oplus A$ by Corollary 7.16. The identity spans $N^{\prime} \cap M_{1}$ modulo $\mathbb{C} c_{P} \oplus A \oplus B$.

With the results so far, it is easy to see that all the structure constants for multiplication are determined: multiplication of any basis element by $e_{N}$ produces $e_{N}$; multiplication within $\mathfrak{A}$ is determined by Corollaries 7.11 and 5.7. Similarly multiplication within $\mathfrak{B}$ is determined by Corollaries 7.15 , 7.16] and the explicit form of $1_{A}$ in Corollary 7.12. This leaves only multiplication between $\mathfrak{A}$ and $\mathfrak{B}$. But $e_{P Q} e_{P}=e_{P}$ (and other versions with $P$ and $Q$ interchanged) takes care of this. Note also that $\mathfrak{C}=\mathfrak{C}^{*}$ so that the $*$-algebra structure of $N^{\prime} \cap M$ is explicitly determined on the basis $\mathfrak{C}$.

We now turn to comultiplication. The $*$ structure for comultiplication is rotation by $\pi$ and insertion of $*$ 's of elements. Inspection shows that the basis $\mathfrak{C}$ is stable under this operation since $u=u^{*}$ is a projection for comultiplication by Corollary 7.5. The subsets $\mathfrak{A}$ and $\mathfrak{B}$ no longer correspond to the algebraic structure, but it will be convenient to organise the calculation according to them. Determination of all the structure constants will just be a long sequence of cases, the most difficult of which will be diagrammatic and make frequent use of Lemma 7.3. Note that the shading of the picture will be the opposite of that in Lemma 7.3 since $u$ is in $M_{1}$ and not in $M_{2}$. Occasionally the diagrammatic reductions will produce the element $u$ itself. It is easy to express $u$ as a linear combination of basis elements since $u\left(1-e_{N}-1_{A}-1_{B}\right)=1-e_{N}-1_{A}-1_{B}$ and $u$ times any element of $\mathfrak{A} \cup \mathfrak{B}$ is another element of $\mathfrak{A} \cup \mathfrak{B}$.

We will also use the exchange relation for biprojections from [2]:
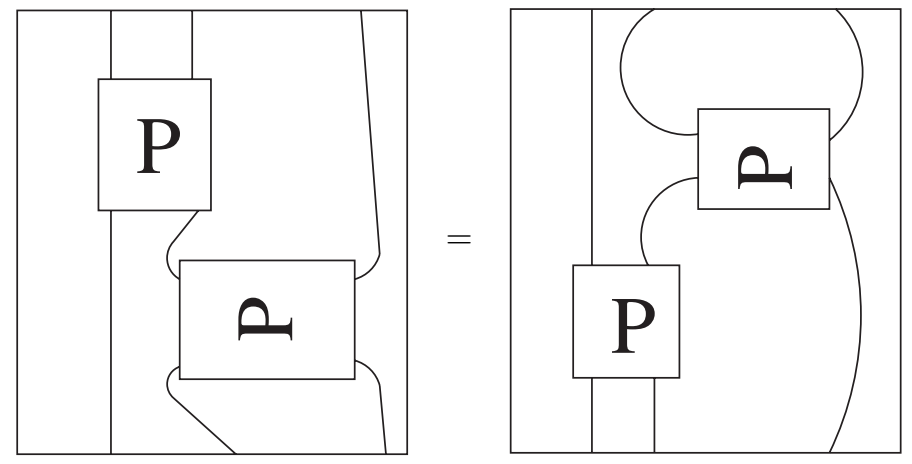
We have no need for the exact values of the structure constants; we only need to know that they could be calculated explicitly. Thus we introduce the notation $x \approx y$ to mean that the elements $x$ and $y$ of $N^{\prime} \cap M_{1}$ are equal up to multiplication by a constant that could be calculated explicitly.

Thus for instance $e_{N} \approx \tilde{1}$ when $\tilde{1}$ is the identity for comultiplication. So all structure constants for comultiplication by $e_{N}$ are determined. Comultiplication by 1 is easy by the formula $x \circ 1 \approx \operatorname{tr}(x) 1$ for $x \in N^{\prime} \cap M_{1}$, and the only trace that requires any work at all is that of $u e_{P Q}$, which is determined from Corollaries 7.15 and 7.16 .

Case 1. Comultiplication within $\mathfrak{A}$. We may replace $u e_{P}$ by $e_{Q} e_{P}$ which is $\approx$ the projection onto $L^{2}(P Q)$ for comultiplication. It is thus greater than $e_{P}$ and $e_{Q}$, so $e_{P} \circ\left(e_{P} e_{Q}\right) \approx e_{P}$. The first case where any work is required is $\left(u e_{P}\right) \circ\left(u e_{Q}\right)$ and up to simple modifications of the argument this handles all comultiplications within $\mathfrak{A}$. The labelled tangle defining $\left(u e_{P}\right) \circ\left(u e_{Q}\right)$ is:

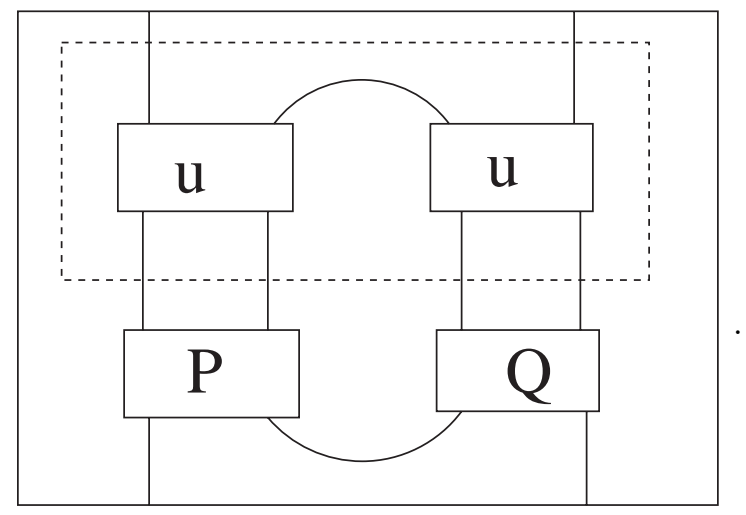

Applying Lemma 7.3 to the region inside the dotted rectangle we obtain:

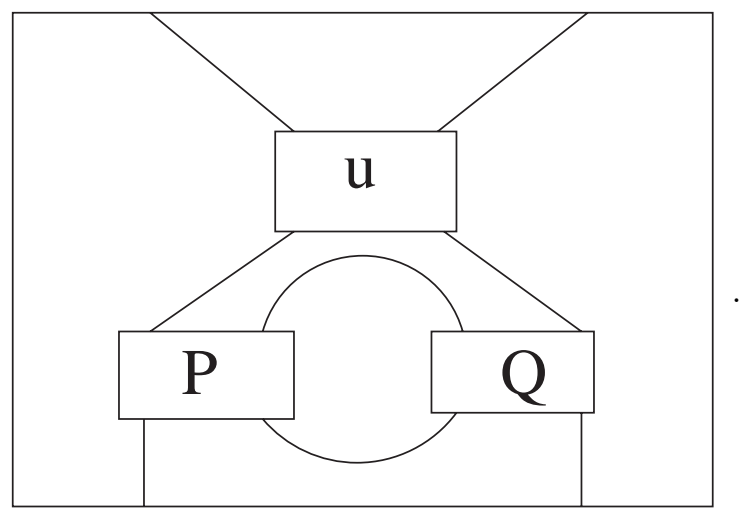

But this is $\approx e_{P Q} u$, which is a basis element.

Case 2. Comultiplication within $\mathfrak{B}$. Comultiplying $e_{P Q}$ with itself or with $e_{Q P}$ is easy since under comultiplication $e_{P}$ and $e_{Q}$ generate a $2 \times 2$ matrix algebra mod 1 and $e_{P} \circ e_{Q} \approx e_{P Q}$. Comultiplying $e_{P Q}$ or $e_{Q P}$ with $u e_{P Q}$ or $u e_{P Q}$ can, after 
applying Corollary 7.10 if necessary, yield a labelled tangle like:

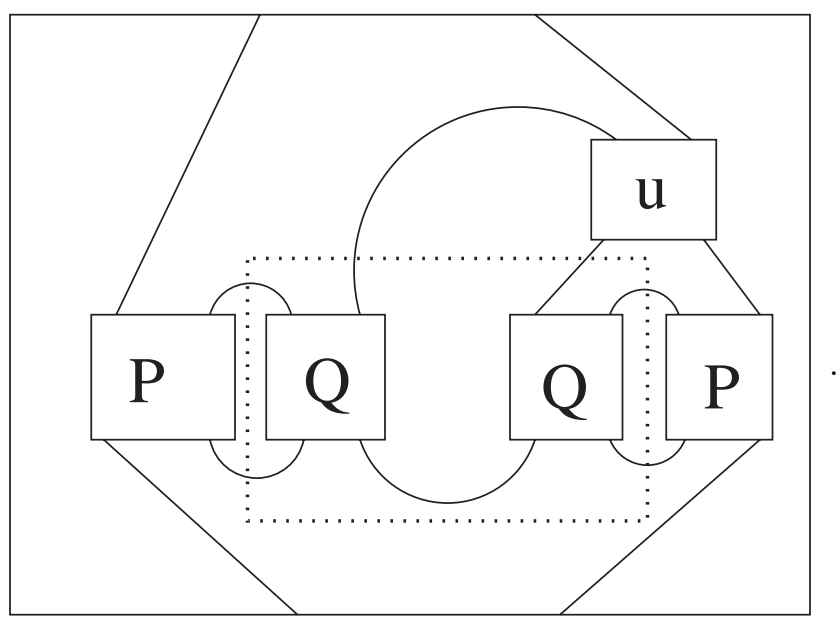

The point of using Corollary 7.10 is to ensure that in the dotted rectangle we see either two $P$ 's or two $Q$ 's. The $u$ may thus end up below the $P$ 's and $Q$ 's but that does not affect the rest of the argument. In the dotted rectangle we may thus apply the exchange relation for $Q$ to obtain, after a little isotopy:

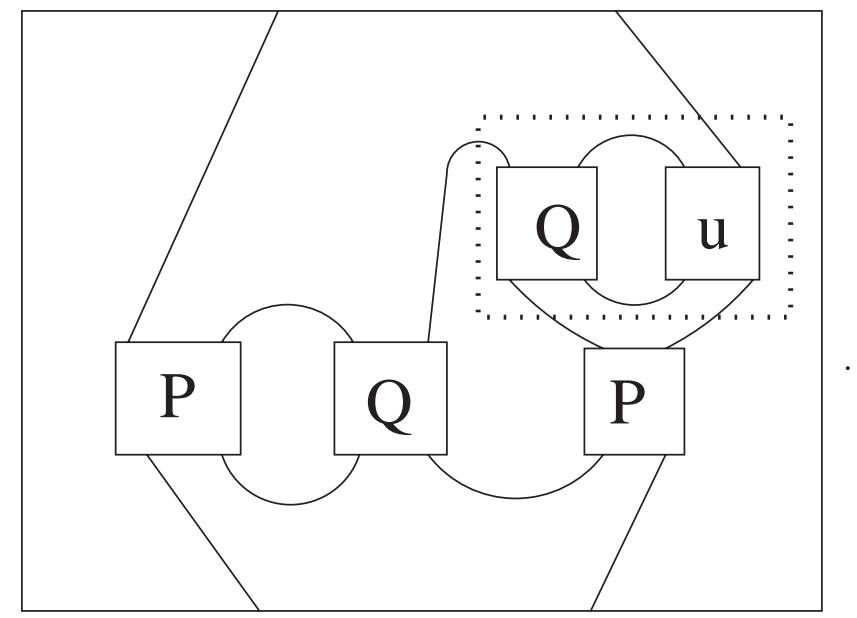

Notice that inside the dotted rectangle we see the comultiplication of $e_{Q}$ and $u$. Replacing $u$ by $2 e_{R}-1$ gives 2 tangles, the one with the identity being $\approx e_{P} \circ e_{Q} \circ e_{P}$. The tangle with $e_{R}$ can be handled easily since $e_{Q} \circ e_{R}=1$, which also yields $e_{P} \circ e_{Q} \circ e_{P}$.

Finally, we need to be able to comultiply $u e_{P Q}$ with itself and $u e_{Q P}$. This is done very much like comultiplying $u e_{P}$ and $u e_{Q}$ except that after applying Lemma 7.3 we find a coproduct of more than two terms on $e_{P}$ and $e_{Q}$. These words may be reduced to $e_{P}, e_{Q}, e_{P Q}$ or $e_{Q P}$ modulo $e_{N}$. The term with $e_{N}$ will produce a $u$ by itself, but as observed above we know how to write $u$ as an explicit linear combination of basis elements.

Case 3. Comultiplication between $\mathfrak{A}$ and $\mathfrak{B}$. Terms without $u$ like $e_{P} \circ e_{P Q}$ are simple. The most difficult case is of the form $e_{P} \circ u e_{Q P}$, but as above we may 
rearrange it so that there are two like terms in the dashed rectangle below:

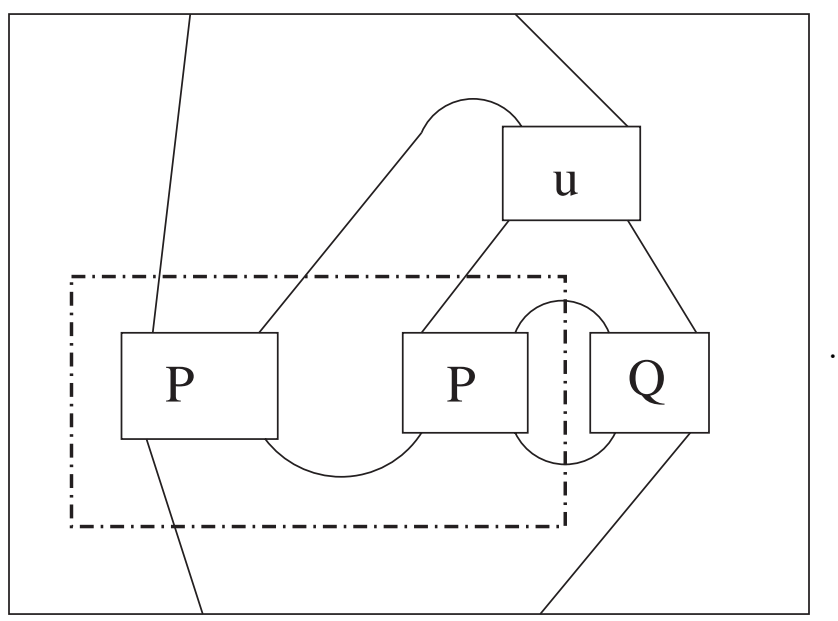

Applying the exchange relation as before we obtain:

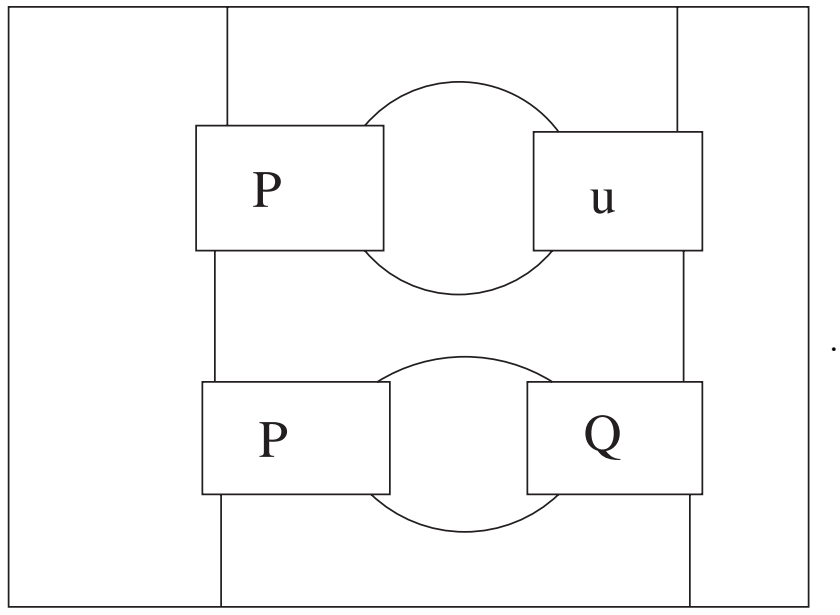

Note the comultiplication of $u$ and $e_{P}$ which can be reduced to an explicit linear combination of basis elements using $u=2 e_{R}-1$ and $e_{R} \circ e_{P} \approx 1$.

The coproduct of $u e_{P}$ with $e_{P Q}$ works similarly except that applying the exchange relation immediately produces an explicit multiple of a basis element. Finally, terms like $u e_{P} \circ u e_{P Q}$ can be reduced to explicit linear combinations of basis elements using Lemma 7.3 and comulitplication of words on $e_{P}$ and $e_{Q}$. Once again $u$ terms may be produced.

Lemma 7.19. Let $v \in M^{\prime} \cap M_{2}$ be the self-adjoint unitary in the normaliser of $M_{1}$ guaranteed by the form of the dual principal graph in Theorem 5.8. Then $v A v=B$. 
Proof. By Figure 7.4 we have

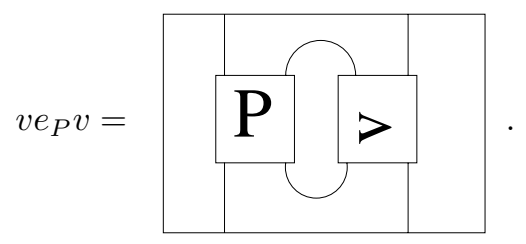

So

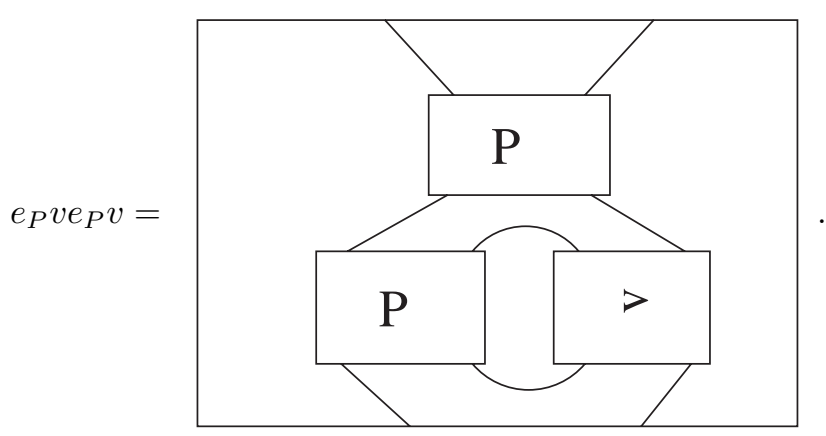

Applying the exchange relation to this we obtain

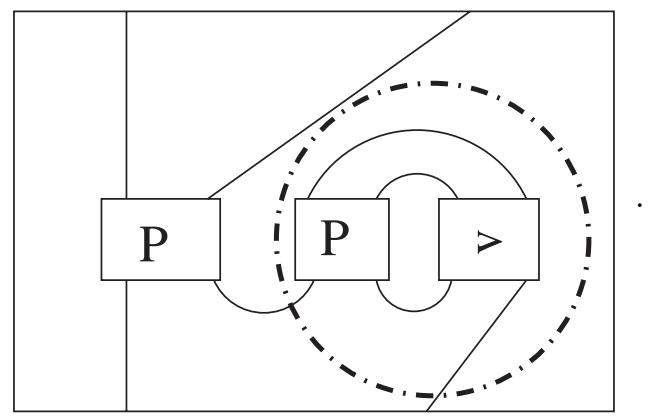

Inside the dashed circle we recognise a multiple of the trace in $M_{2}$ of the product in $M^{\prime} \cap M_{2}$ of the projection $e_{\bar{P}}$ defined by $e_{P}$ and $v$. But $v$ bears the same relation to this coprojection as $u$ does to $e_{P}$, so by Lemma 7.8 we obtain zero. Thus $e_{P} V e_{P} V=0$. We may apply Corollary 7.10 to $e_{\bar{P}}$ and $v$ to deduce in the same way that $e_{Q} V e_{P} V=0$. This is enough to conclude that $v A v=B$ from the structure of $N^{\prime} \cap M_{1}$ which is normalised by $v$.

Corollary 7.20. If $e_{M}$ is the projection onto $L^{2}(M)$ in the basic construction of $M_{2}$ from $M_{1}$, then $\mathfrak{D}=\mathfrak{C} e_{M} \mathfrak{C} \cup \mathfrak{A} \cup v \mathfrak{A} \cup \mathfrak{B} \cup v \mathfrak{B}$ is a basis for $N^{\prime} \cap M_{2}$.

Proof. From the principal graph, $N^{\prime} \cap M_{2}$ is the direct sum of the ideal $\mathfrak{I}$ generated by $e_{M}$, which is isomorphic to a basic construction coming from the pair $N^{\prime} \cap$ $M \subseteq N^{\prime} \cap M_{1}$, and a $4 \times 4$ matrix algebra. Since $N \subseteq M$ is irreducible the map $x \otimes y \mapsto x e_{M} y$ is a vector space isomorphism from $N^{\prime} \cap M_{1} \otimes N^{\prime} \cap M_{1}$ to $\mathfrak{I}$. Thus $\mathfrak{C} e_{M} \mathfrak{C}$ is a basis for $\mathfrak{I}$.

Since $v$ is in the normaliser of $M_{1}$, it is orthogonal to $M_{1}$ by irreducibility and $N^{\prime} \cap$ $M_{2}$ contains a copy of the crossed product of $N^{\prime} \cap M_{1}$ by the period 2 automorphism given by $A d v$. By the previous lemma the algebra generated by $A, B$, and $v$ is a $4 \times 4$ matrix algebra - call it $\mathfrak{E}$. It is spanned modulo $\mathfrak{I}$ by $\mathfrak{A} \cup v \mathfrak{A} \cup \mathfrak{B} \cup v \mathfrak{B}$ since $A$ 
and $B$ are spanned modulo $e_{N}$ by $\mathfrak{A}$ and $\mathfrak{B}$ respectively (see the proof of Theorem (7.18). Since a matrix algebra is simple, to check that $\mathfrak{E}$ spans $N^{\prime} \cap M_{2} \bmod \mathfrak{I}$ we need only show that it is not contained in $\mathfrak{I}$. But from the principal graph we see that $A$ itself is nonzero mod $\mathfrak{I}$.

7.5. The uniqueness proof and some corollaries. We can now give the main argument for the uniqueness of a subfactor of index $(2+\sqrt{2})^{2}$ with noncommuting intermediate subfactors. It relies on the "exchange relation" developed by Landau in [27. We begin with a planar algebra result from which our uniqueness will follow.

NOTE: We will assume that all planar algebras $P$ satisfy $\operatorname{dim} P_{1}=1$.

Definition 7.21. Let $P=P_{n}$ be a planar algebra and $\mathfrak{R}$ a self-adjoint subset of $P_{2}$. Let $\mathfrak{Y}$ be the set of planar 3 -tangles labelled with elements of $\mathfrak{R}$, with at most one internal disc. We say that $\mathfrak{R}$ satisfies an exchange relation if there are constants $b_{Q, R, Y}, c_{Q, R, S, T}$ and $d_{Q, R, S, T}$ such that
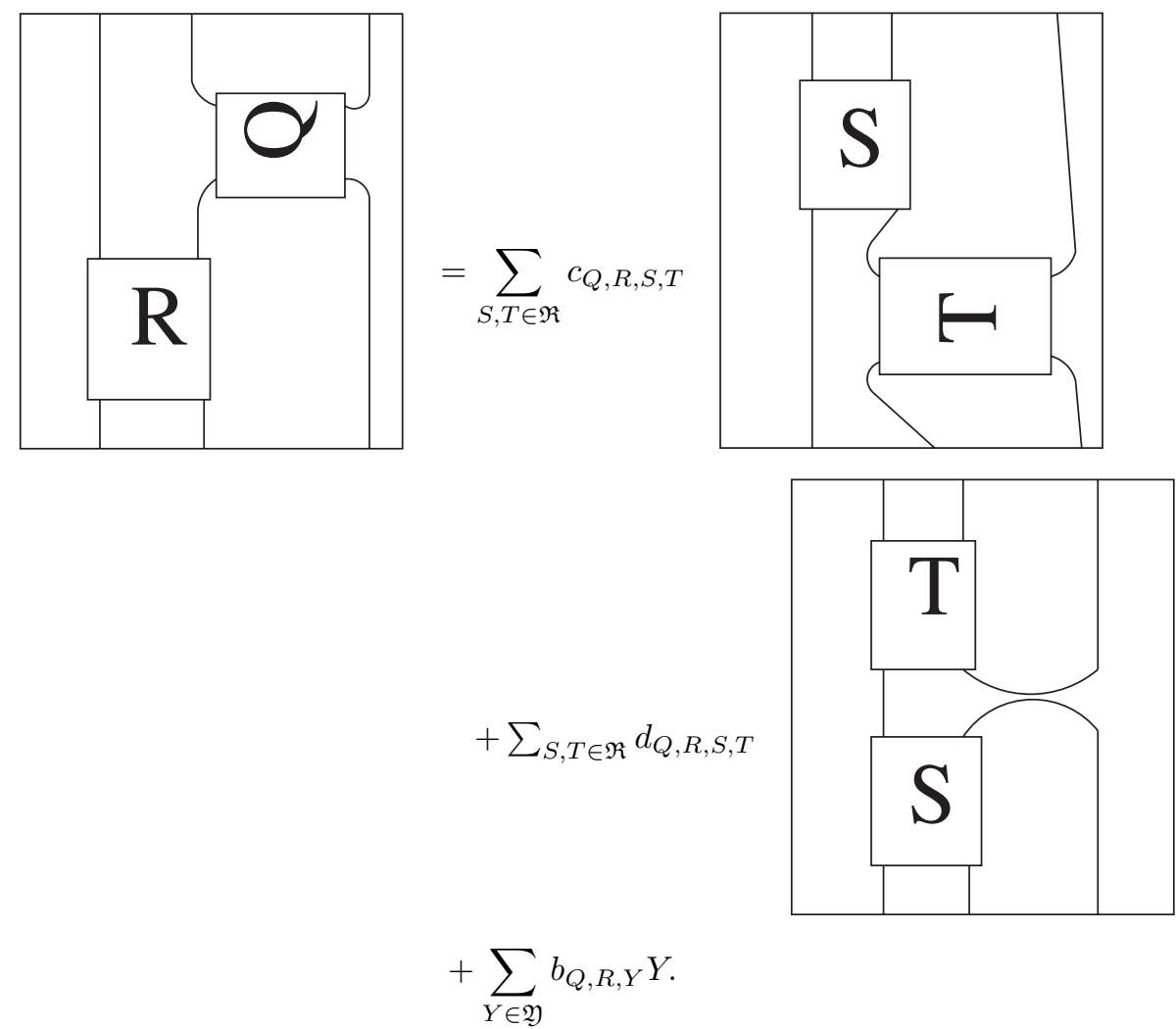

The constants will be called the exchange constants for $\mathfrak{R}$.

Theorem 7.22 (Landau, 27]). A subfactor planar algebra $P$ generated by $\mathfrak{R}=$ $\mathfrak{R}^{*} \subseteq P_{2}$ is determined up to isomorphism by the exchange constants for $\mathfrak{R}$ and the traces and cotraces of elements in $\Re$.

The idea of the proof is that one may calculate the partition function of any labelled tangle in $P_{0}$ by applying the exchange relation. The strategy is to take any face and reduce it to a bigon, which is either a multiplication or comultiplication of 
elements in $\Re$. But multiplication and comultiplication are also determined by the exchange relation by suitably capping off the pictures in the above definition. As soon as the planar algebras in question are nondegenerate in the sense that they are determined by the partition functions of labelled planar tangles in $P_{0}$, the theorem will hold. The isomorphism between two planar algebras with the same subset $\mathfrak{R}$ is defined by extending the identity map from $\mathfrak{R}$ to itself to all labelled tangles on $\mathfrak{R}$. Then any relation for one planar algebra is necessarily a relation for the other by the nondegenerate property of the partition function as a bilinear/sesquilinear form on the $P_{n}$. This strategy for proving uniqueness was already used for a proof of the uniqueness of the $E_{6}$ and $E_{8}$ subfactors in [21.

Lemma 7.23. Let $P$ be a subfactor planar algebra with $\mathfrak{R}$ a self-adjoint subset of $P_{2}$ which satisfies an exchange relation. Then the exchange constants for $\mathfrak{R}$ are determined by the traces and cotraces of elements of $\mathfrak{R}$ together with the structure constants for multiplication and comultiplication of elements of $\Re$.

Proof. Using positive definiteness of the inner product given by the trace on $P_{3}$, it suffices to prove that the partition function of any planar diagram with at most 4 internal discs, all labelled with elements of $\mathfrak{R}$, is determined by the given structure constants.

For this, we may suppose that the labelled diagrams are connected and by our hypothesis on $\operatorname{dim} P_{1}$, we may suppose that no 2-box is connected to itself. If there are 4 internal discs, one must be connected to another with a multiplication or a comultiplication. This reduces us to the case of 3 internal boxes where it is even clearer. To see these assertions it is helpful to view the labelled tangles as the generic planar projections of links in $\mathbb{R}^{3}$ which are obtained by shrinking the internal 2-boxes to points.

Putting the previous results together we have:

Theorem 7.24. Let $N_{1} \subseteq M_{1}$ and $N_{2} \subseteq M_{2}$ be two irreducible $\mathrm{II}_{1}$ subfactors of index $(2+\sqrt{2})^{2}$ with pairs $P_{1}, Q_{1}$ and $P_{2}, Q_{2}$ of noncommuting intermediate subfactors of index $2+\sqrt{2}$. Then there is a unique isomorphism from the planar algebra for $N_{1} \subseteq M_{1}$ to the planar algebra of $N_{2} \subseteq M_{2}$ which extends the map sending $e_{P_{1}}$ and $e_{Q_{1}}$ to $e_{P_{2}}$ and $e_{Q_{2}}$ respectively.

Proof. The only allowed principal graph for the elementary subfactors is $A_{7}$. So there is no extra structure, and we know the principal graph and dual principal graph. The normalising unitaries $u_{i}, i=1,2$ can be written as an explicit linear combination of $e_{N}, 1$ and products and coproducts of $e_{P_{i}}$ and $e_{Q_{i}}$. Then form the sets $\mathfrak{A}_{i}$ and $\mathfrak{B}_{i}, i=1,2$ in the obvious way. The planar algebra for $N_{i} \subseteq M_{i}$ is generated by $\mathfrak{A}_{i}$ and $\mathfrak{B}_{i}$ by Corollary 7.20 , By Theorem 7.18 and Lemma 7.23 we may apply Theorem 7.22 to the sets $\mathfrak{R}_{i}=\mathfrak{A}_{i} \cup \mathfrak{B}_{i}$ to deduce the result. (The traces and cotraces of the basis elements of $\mathfrak{C}$ were determined in the course of proving Theorem 7.18)

Corollary 7.25. Given a quadrilateral $N \subset P, Q \subset M$ with $[M: N]=6+4 \sqrt{2}$ and such that $P$ and $Q$ do not commute, there are further subfactors $\tilde{P}$ and $\tilde{Q}$ with $[M: \tilde{P}]$ and $[M: \tilde{Q}]$ equal to $2+\sqrt{2}$, which commute with both $P$ and $Q$ and are at an angle $\cos ^{-1}(\sqrt{2}-1)$ to each other.

Proof. This is the case for the example, so by uniqueness it is always true. 
It is obvious that the projections onto $\tilde{P}$ and $\tilde{Q}$ are in $B \bmod e_{N}$.

Corollary 7.26. The only subfactors between $N$ and $M$ are $P, Q, \tilde{P}, \tilde{Q}, R$ and $S$, so the intermediate subfactor lattice is

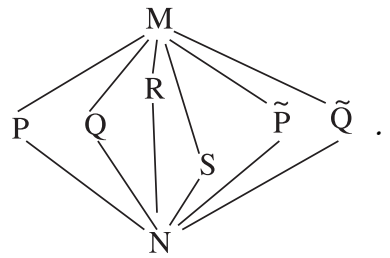

Proof. Let $T$ be a seventh intermediate subfactor. From the principal graph and obvious index restrictions the possible values of $(6+4 \sqrt{2}) \operatorname{tr}\left(e_{T}\right)$ are $2+\sqrt{2}, 3+2 \sqrt{2}$ and 2 . The cases $3+2 \sqrt{2}$ and 2 correspond to index 2 subfactors and would show up as extra vertices on either the dual or dual principal graphs, so we must have $\operatorname{tr}\left(e_{T}\right)=1 /(2+\sqrt{2})$. This forces $e_{T}-e_{N}$ to be a minimal projection in either $A$ or $B$, so by the previous corollary and the observation after it we may suppose wolog that $e_{T}-e_{N} \in A$. If $e_{P} e_{T}=e_{N}$, then by a $2 \times 2$ matrix calculation, $T$ makes a forbidden angle with $Q$. So the angle between all three of $P, Q$ and $T$ is $\cos ^{-1}(\sqrt{2}-1)$. But by Lemma 7.7 applied to $T, T$ and $R$ do not commute. So there must be a fourth subfactor $\alpha(T)$ which makes the same angle with all of $P, Q$, and $T$. By Lemma 7.9 this is not allowed. This contradicts the existence of $T$.

Corollary 7.27. If $M$ is hyperfinite, then there is an automorphism of $M$ sending $P$ to $\tilde{P}$ and $Q$ to $\tilde{Q}$.

Proof. This follows from Theorem 7.24] and Popa's classification theorem 31, which states that in finite depth one may construct the subfactor directly as the completion of the inductive limit of the tower of relative commutants.

It is not obvious what the automorphism of the previous corollary looks like in the GHJ realisation of section 6. It will certainly require the complex numbers to write it down as guaranteed by the next result. Observe first that the $D_{5}$-based GHJ example of Figure 6.4 is defined over the real numbers, so the intermediate subfactors exist in the setting of real $\mathrm{II}_{1}$ factors. That the GHJ pair for $D_{5,2}$ needs the complex numbers is the next result.

Corollary 7.28. If $N \subset P, Q \subset M$ is a noncommuting quadrilateral of real $\mathrm{II}_{1}$ factors with $[M: N]=6+4 \sqrt{2}$, then $P$ and $Q$ are the only intermediate subfactors of index $2+\sqrt{2}$.

Proof. Let $N \subset M$ be the subfactor for the $D_{5,2}$ Coxeter graph. Since this subfactor may be defined over the reals (as the GHJ subfactor for the trivalent vertex), complex conjugation defines a conjugate linear *-automorphism $\sigma$ of $N \subset M$ with $\sigma(\tilde{P})=\tilde{P}$ and $\sigma(\tilde{Q})=\tilde{Q}$ but with $\sigma\left(g_{i}\right)=g_{i}^{*}$, so $\sigma(P)=Q$. Thus $\sigma$ will act on the planar algebra of $N \subset M$ exchanging $e_{P}$ and $e_{Q}$. However, the fixed points for $\sigma$ acting on the planar algebra form again a planar algebra. So there is a real subfactor $N_{\mathbb{R}} \subset M_{\mathbb{R}}$ with $\left[M_{\mathbb{R}}: N_{\mathbb{R}}\right]=6+4 \sqrt{2}$ having a pair $\left(\tilde{P}^{\sigma}\right.$ and $\left.\tilde{Q}^{\sigma}\right)$ of noncommuting intermediate subfactors of index $2+\sqrt{2}$ and no other intermediate subfactors of the same index since $\sigma\left(e_{P}\right)=e_{Q} \neq e_{P}$. Our uniqueness result never used the complex numbers (all the structure constants were real) so that no other such real subfactor can have more than two intermediate subfactors of index $2+\sqrt{2}$. 


\section{REFERENCES}

1. Birman, J. (1974). Braids, links and mapping class groups. Annals of Mathematical Studies, 82. MR 0375281 (51:11477)

2. Bisch, D. (1994). A note on intermediate subfactors. Pacific Journal of Mathematics, 163, 201-216. MR 1262294 (95c:46105)

3. Bisch, D. (1997). Bimodules, higher relative commutants and the fusion algebra associated to a subfactor. In Operator algebras and their applications. Fields Institute Communications, Vol. 13, American Math. Soc., 13-63. MR.1424954 (97i:46109)

4. Bisch, D. and Jones, V. F. R. (1997). Algebras associated to intermediate subfactors. Inventiones Mathematicae, 128, 89-157. MR1437496 (99c:46072)

5. Bisch, D. and Jones, V. F. R. (1997). A note on free composition of subfactors. In Geometry and Physics, (Aarhus 1995), Marcel Dekker, Lecture Notes in Pure and Applied Mathematics, Vol. 184, 339-361. MR:1423180 (97m:46102)

6. Bisch, D. and Jones, V. F. R. (2000). Singly generated planar algebras of small dimension. Duke Mathematical Journal, 101, 41-75. MR:1733737 (2002f:46118)

7. Bisch, D. and Jones, V. F. R. (2003). Singly generated planar algebras of small dimension. II. Advances in Mathematics, 175, 297-318. MR.1972635 (2004d:46073)

8. Bratteli, O. (1972). Inductive limits of finite dimensional $C^{*}$-algebras. Transactions of the American Mathematical Society, 171, 195-234. MR0312282 (47:844)

9. Connes, A. (1976). Classification of injective factors. Annals of Mathematics, 104, 73-115. MR0454659 (56:12908)

10. Evans, D. E. and Kawahigashi, Y. (1998). Quantum symmetries on operator algebras. Oxford University Press. MR:1642584 (99m:46148)

11. Goldman, M. (1960). On subfactors of type $\mathrm{II}_{1}$. The Michigan Mathematical Journal, 7, 167-172. MR0107827 (21:6549)

12. Goodman, F., de la Harpe, P. and Jones, V. F. R. (1989). Coxeter graphs and towers of algebras. MSRI Publications (Springer), 14. MR0999799(91c:46082)

13. Izumi, M. (1991). Application of fusion rules to classification of subfactors. Publications of the RIMS, Kyoto University, 27, 953-994. MR1145672 (93b:46121)

14. Izumi, M. and Kosaki, H. (2002). On a subfactor analogue of the second cohomology. Reviews in Mathematical Physics, 14, 733-757. MR1932664 (2004a:46061)

15. Jones, V. F. R. (1980). Actions of finite groups on the hyperfinite type $\mathrm{II}_{1}$ factor. Memoirs of the American Mathematical Society, 237. MR0587749 (81m:46094)

16. Jones, V. F. R. (1983). Index for subfactors. Inventiones Mathematicae, 72, 1-25. MR.0696688 (84d:46097)

17. Jones, V. F. R. (1985). A polynomial invariant for knots via von Neumann algebras. Bulletin of the American Mathematical Society, 12, 103-112. MR0766964 (86e:57006)

18. Jones, V. F. R. (1989). On knot invariants related to some statistical mechanical models. Pacific Journal of Mathematics, 137, 311-334. MR0990215 (89m:57005)

19. Jones, V. F. R. (in press). Planar algebras I. New Zealand Journal of Mathematics. arXiv.math.QA/9909027.

20. Jones, V. F. R. (2000). The planar algebras of a bipartite graph. in Knots in Hellas '98, World Scientific, 94-117. MR.1865703 (2003c:57003)

21. Jones, V. F. R. (2001). The annular structure of subfactors. in Essays on geometry and related topics, Monographies de L'Enseignement Mathematique, 38, 401-463. MR1929335 (2003j:46094)

22. Jones, V. F. R. (1994) On a family of almost commuting endomorphisms. Journal of functional analysis, 119, 84-90. MR.1274584 (95c:46106)

23. Jones, V. F. R. (2003) Quadratic tangles in planar algebras. In preparation: http://math.berkeley.edu/ vfr/.

24. Jones, V. F. R. and Sunder, V. S. (1997). Introduction to subfactors. London Math. Soc. Lecture Notes Series 234, Cambridge University Press. MR 1473221 (98h:46067)

25. Jones, V. F. R. and Xu, F. (2004). Intersections of finite families of finite index subfactors. International Journal of Mathematics, 15, 717-733. MR2085101 (2005g:46118)

26. Landau, Z. (2001). Fuss-Catalan algebras and chains of intermediate subfactors. Pacific Journal of Mathematics, 197, 325-367. MR1815260(2002h:46103) 
27. Landau, Z. (2002). Exchange relation planar algebras. Journal of Functional Analysis, 195, $71-88$.

28. Ocneanu, A. (1991). Quantum symmetry, differential geometry of finite graphs and classification of subfactors, University of Tokyo Seminary Notes 45, (Notes recorded by Kawahigashi, Y.).

29. Okamoto, S. (1991). Invariants for subfactors arising from Coxeter graphs. Current Topics in Operator Algebras, World Scientific Publishing, 84-103. MR1193932 (94c:46127)

30. Pimsner, M. and Popa, S. (1986). Entropy and index for subfactors. Annales Scientifiques de l'École Normale Supérieure, 19, 57-106. MR0860811 (87m:46120)

31. Popa, S. (1990). Classification of subfactors: Reduction to commuting squares. Inventiones Mathematicae, 101, 19-43. MR1055708(91h:46109)

32. Popa, S. (1995). An axiomatization of the lattice of higher relative commutants of a subfactor. Inventiones Mathematicae, 120, 427-446. MR1334479 (96g:46051)

33. Sano, T. and Watatani, Y. (1994). Angles between two subfactors. Journal of Operator Theory, 32, 209-241. MR 1338739 (96j:46062)

34. Sauvageot, J. L. (1983). Sur le produit tensoriel relatif d'espace de Hilbert. Journal of Operator Theory, 9, 237-252. MR0703809 (85a:46034)

35. Temperley, H. N. V. and Lieb. E. H. (1971). Relations between the "percolation" and "colouring" problem and other graph-theoretical problems associated with regular planar lattices: Some exact results for the "percolation" problem. Proceedings of the Royal Society A, 322, 251-280. MR0498284 (58:16425)

36. Watatani, Y. (1996). Lattices of intermediate subfactors. Journal of Functional Analysis, 140, 312-334. MR1409040 (98c:46134)

Department of Mathematics, University of California at Berkeley, Berkeley, CalIFORNIA 94720

E-mail address: pinhas@math.berkeley.edu

Department of Mathematics, University of California at Berkeley, Berkeley, CalIFORNIA 94720

E-mail address: vfr@math.berkeley.edu 